$\mathrm{PM} / 01-71$

\title{
SUSY Scalar Production in the Electroweak Sudakov Regime of Lepton Colliders *
}

\author{
M. Beccaria ${ }^{a, b}$, M. Melles ${ }^{c}$, F.M. Renard ${ }^{d}$ and C. Verzegnassi ${ }^{e, f}$ \\ ${ }^{a}$ Dipartimento di Fisica, Università di Lecce \\ Via Arnesano, 73100 Lecce, Italy. \\ ${ }^{b}$ INFN, Sezione di Lecce \\ ${ }^{c}$ Paul Scherrer Institute (PSI), CH-5232 Villigen, Switzwerland \\ ${ }^{d}$ Physique Mathématique et Théorique, UMR 5825 \\ Université Montpellier II, F-34095 Montpellier Cedex 5. \\ e Dipartimento di Fisica Teorica, Università di Trieste, \\ Strada Costiera 14, Miramare (Trieste) \\ ${ }^{f}$ INFN, Sezione di Trieste
}

\begin{abstract}
We consider the production of SUSY scalar pairs at lepton colliders, for c.m. energies much larger than the mass of the heaviest SUSY (real or virtual) particle involved in the process. In that energy regime, we derive the leading and subleading terms of the electroweak Sudakov logarithms in the MSSM, first working at one loop with physical states and then resumming to all orders with asymptotic expansions. We show that the first order of the resummed expression reproduces the physical one loop approximation, and compute systematically the possible effects on various observables both at one loop and to all orders. We discuss the regimes and the observables where the one loop approximation can or cannot be trusted, working in an energy range between $1 \mathrm{TeV}$ and $4 \mathrm{TeV}$ under a "light" SUSY mass assumption. As a byproduct of our analysis, we propose a determination of the MSSM parameter $\tan \beta$ showing how a relative accuracy $\simeq 25 \%$ can be easily achieved in the region $\tan \beta \gtrsim 14$, under reasonable experimental assumptions.
\end{abstract}

PACS numbers: 12.15.-y, 12.15.Lk, 14.80.Ly, 14.80.Cp

Typeset using REVTEX

*Partially supported by EU contract HPRN-CT-2000-00149 


\section{INTRODUCTION}

The fact that the process of electron-positron annihilation into a Standard Model (SM) fermion pair is dominated at high energy by large electroweak logarithms of "Sudakov-type" [1] has become nowadays completely established. After the first numerical calculations at one loop, that evidentiated the unexpectedly large size of the leading quadratic (DL) and subleading linear [2.3] (SL) terms, a great amount of theoretical work has been devoted to the task of computing this type of effect beyond the one-loop order. This hard calculation becomes imperative if one wants to provide a safe theoretical description of the process for c.m. energies in the $\mathrm{TeV}$ range, where the truncation at one loop of the perturbative expansion would be certainly unreliable [2].

In general, one can say that two types of approaches have been followed. The first one is based on the study of the process in a suitable asymptotic energy region, where either technical or theoretical simplifications are supposed to be valid. Although this definition is not compulsory, we shall refer to this energy range as that where an "electroweak Sudakov regime" has settled. In this range, a resummation of the Sudakov logarithms to all orders has been proposed by different groups [4]. For the specific case of a final fermion pair production, the results seem nowadays to agree, as thoroughly discussed in a very recent paper [5], and for a detailed comparison we defer to the existing literature [4:67.

In the second approach, one has tried to make explicit calculations of the Sudakov effects at two loops, working with "physical" (i.e. not asymptotic) processes and computing the high energy limits of the derived expressions [7]. This second approach clearly provides a very important test of the reliability of the first one, by comparison of the corresponding two-loop contributions. At the moment, this comparison has been successful for the leading logarithmic terms of the asymptotic expansion. For the subleading terms, which depend both on the c.m. energy $\sqrt{q^{2}}$ and the c.m. scattering angle $\theta$, a clean high energy resummation prescription now exists [5], but the corresponding "physical" two-loop calculation is not yet available. In spite of this, a fair conclusion is that, at the moment, the electroweak Sudakov logarithms in the process of electron - positron annihilation are under control, provided that the simple one-loop expansion is replaced, in the $\mathrm{TeV}$ c.m. energy range, by a more complete calculation. To be more precise, it should be stressed, though, that the necessity of this replacement is strongly dependent on the considered observable and energy, and that for a special set of quantities (like, e.g., forward-backward asymmetries) the validity of a one loop expansion might be still acceptable at realistic collider energies. For an exhaustive discussion of this point we defer to a very recent paper [8].

An important question in this subject is that of when the "electroweak Sudakov regime" starts. Otherwise stated, at which energy can one assume that an expansion of Sudakov type provides a "satisfactory" description of the various processes, i.e. one that reproduces the relevant experimental observables at the requested theoretical accuracy? In [3], a tentative one loop analysis was prepared by fitting the numerical values at variable energy of various quantities, rigorously computed, with a logarithmic expansion "à la Sudakov" that included, beyond the leading and the sub-leading terms (including the RG ones) an unknown constant, to be fitted. The result showed that, in the SM, an "electroweak Sudakov regime" was actually settling when the c.m. energy approached the typical value $\sqrt{q^{2}}=1 \mathrm{TeV}$, since the fitted parameters of the logarithms where exactly the theoretical Sudakov ones. It also 
showed that, at such energies, the numerical value of the constant term, although smaller than those of the various logarithms, was not negligible at the typical level of accuracy of a relative one percent (to be assumed from now on as the aimed experimental reach of future lepton colliders). The conclusion thus seems to be that, within the SM, electron - positron annihilation appears to enter a "Sudakov regime" for c.m. energies of the TeV size to be hopefully reached by a future LC [9] or CLIC [10] experiments.

As a remark that appears, at this stage, somewhat formal, it can be finally noticed that the knowledge of the Sudakov logarithms (and, also, of the well known and precisely determined RG ones [11]) would be sufficient to predict the slope (i.e. the variation with energy) of each experimental observables in its "Sudakov regime" since clearly, in this calculation, the constant terms will disappear. Within the SM, this procedure would not provide particularly interesting consequences, although it could be always considered a valuable overall test of the hard theoretical calculations.

All the previous statements and conclusions are strictly valid, as we said, in the SM theoretical framework. A spontaneous question that arises at this point is that of whether the obtained picture remains valid in a theoretical extension of the SM, for which the same type of perturbative expansion is supposed to be valid. A rather natural first candidate of this kind seems to be the minimal supersymmetric extension of the SM, the MSSM. Analogously, the rather natural processes to be considered seem to be the same ones that we have previously listed, for which the Sudakov effect in the SM has been fully computed. Here, the extra particles of the MSSM might induce new virtual effects at the one-loop level, thus modifying the coefficients of both the leading and the sub-leading Sudakov logarithms (and, of course, and in an already known way, also those of the RG ones).

An important preliminary problem that arises at this point is that of determining the c.m. energy at which the "SUSY electroweak Sudakov regime" starts. The latter energy is fixed by the request of being sufficiently larger than the mass of the heaviest SUSY particle that appears in the diagrams that generate Sudakov logarithms. Clearly, no precise answer can be given at the moment to this question. In spite of this shortage, one can still proceed in a correct general way by first defining as $M$ the unknown heaviest relevant SUSY mass and computing the Sudakov expansion at asymptotic energies $\sqrt{q^{2}} \gg M$. A next, more speculative step, would be that of assuming a "light $M$ " situation characterized by "reasonable" $M$ values, say below the TeV range. This would allow to make detailed numerical predictions in a supposedly valid SUSY Sudakov regime, that might be easily modified as soon as supersymmetric particles were finally detected.

The first investigation of SUSY Sudakov effects in the process of electron - positron annihilation into a (charged) fermion - anti-fermion pair was recently performed at the perturbative one-loop level [12], and we defer the interested reader to that reference for a detailed discussion of the various considered processes and observables. The two main general results that were derived are the fact that virtual SUSY exchanges in those processes

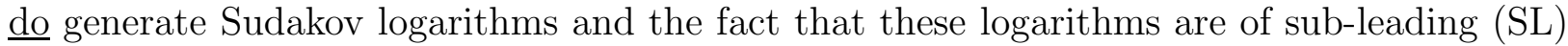
kind and in the generally adopted definition, "universal". At the one loop level, working in the 't Hooft $\xi=1$ gauge, they are only generated by vertex diagrams and not by diagrams of box type. Therefore, they are independent of $\theta$, the c.m. scattering angle. From a formal point of view, they are strictly analogous to the sub-leading logarithms of Yukawa type that arise in the SM for final massive quark production, in practice for production of third family 
quark-antiquark pairs.

For a more detailed numerical description, it was assumed in 12 that the value of the heaviest SUSY mass $M$ was equal to (approximately) a few hundred GeV. Under this assumption, the numerical effects of the SUSY SL at one loop began to be appreciable (say, of a relative few percent) when the c.m. energy was in the $\mathrm{TeV}$ range, in particular they were definitely visible in the supposed CLIC $\left(\sqrt{q^{2}} \simeq 3 \mathrm{TeV}\right)$ region, where in conclusion the virtual effects of the MSSM, under this "light $M$ " working assumption, would be clearly experimentally testable.

An important feature to be clarified at this point is that of which information on certain SUSY parameters can be achieved in this way. In general, the situation is complicated for two reasons: in first place, one does not know exactly the value of $M$; secondly, the role of next-to-sub-leading constant SUSY terms in an asymptotic expansion is unknown (note that, differently from the SM case, a numerical fit of the constant term like that performed in [3] would require the knowledge of all the SUSY parameters that might enter this quantity, and results thus, in practice, hardly performable).

A possible approach that gets rid of the two previous difficulties has been very recently proposed [13]. It is based on the observation that, in the calculation of the slope of experimental observables in a suitable "Sudakov regime", both the constant term and the unknown mass $M$ would disappear, and only the SUSY parameters that enter the coefficient of the Sudakov logarithms would be relevant. In particular, it was shown in 13 that the process of top - antitop production would provide an unconventional way for deriving information, in the MSSM, on the fundamental parameters $\tan \beta$, in a range of values $(20<\tan \beta<40)$ that is very hard to be experimentally explored in other known ways [14, in an energy range $\sqrt{q^{2}} \simeq 3 \mathrm{TeV}$ for a reasonable "light" (i.e. $<500 \mathrm{GeV}$ ) $M$ scenario.

The conclusion of the previous analyses is that, in a c.m. energy range of the TeV size and in a reasonably light $M$ scheme, virtual SUSY effects would play an important role at the future lepton colliders in SM pairs production. An almost obvious attitude is, at this point, that of noticing that, for such $M$ values, direct SUSY pairs production would be copious at such machines, and of asking whether the SUSY virtual effects for these processes would be similar, or "worse", or "better" (i.e. containing more interesting information on parameters) than those of SM pairs creation.

The aim of this first paper is precisely that of analyzing in this spirit the process of scalar (i.e. sfermion of Higgs) SUSY pair production at lepton colliders, and of showing in some detail which relevant information on the MSSM parameters would be obtainable from their virtual effects, and in which possible energy range. Although the final conclusion are supposed to be valid for the special MSSM case, an analysis will be performed in a rather general way, so that our conclusions might be easily generalized for a different type of SUSY model. In this paper, we will try to be as self contained as possible working in a first stage at a "physical" one-loop level, then performing a resummation at sub-leading logarithmic order in an asymptotic regime, showing that the two expansions do coincide at one loop. Having proved this equivalence, we shall perform our numerical analyses in the two different formulations to show the relevance of a logarithmic resummation in the "Sudakov regime" and, in particular, in an energy regime that is within the reach of the (hopefully near) future lepton colliders, with a special emphasis on a possible determination of the fundamental MSSM parameter $\tan \beta$. 
Technically speaking, this paper is organized as follows: Section 2 is devoted to a kinematical description of the processes to be considered, to a definition of their Born observables and to a calculation of their expressions at the one loop level, computed in the asymptotic Sudakov regime, In Section 3, the sub-leading logarithmic order resummation is presented and a comparison with the one-loop expressions of Section 2 is performed. Section 4 contains a discussion of the size of the virtual Sudakov numerical effects, both at one loop and resummed, in a "light $M$ " assumption for a large class of processes. In Section 5 the special role of the $\tan \beta$ dependent Yukawa terms in the MSSM is established and the information derivable on $\tan \beta$ from a measurement of the slopes of certain special processes is exhibited. A final discussion in Section 6 will then conclude the paper.

\section{SUSY SCALAR PRODUCTION AT THE ONE-LOOP LEVEL}

The aim of this Section is that of giving a general description of SUSY scalar pair production at the one loop level, at c.m. energies sufficiently high to justify the use of a logarithmic Sudakov expansion to describe the leading electroweak behavior of the experimental observables. This energy range will be denoted, in our pragmatic definition, as the "electroweak Sudakov regime" of the considered process.

As a first process to be examined, we shall consider that of production of a (charged and neutral) sfermion-antisfermion pair; the treatment of Higgs pair production will then be derivable with simple and straightforward modifications. The considered sfermions will be labelled by their chirality and denoted as $\tilde{f}_{L, R}$. All sleptons and squarks will be considered, with the exception of selectrons. For the latter ones, the theoretical description would be slightly more involved, due to the presence of an extra t-channel exchange, and we shall postpone it to a next dedicated paper [15].

The results obtained for $\tilde{f}_{L, R}$ production will then be easily extended to the case of charged or neutral Higgses.

a) Born level

At the Born level, the process is represented by the photon and $Z$ exchange, depicted in Fig. (1). The corresponding decomposition of the scattering amplitude will be:

$$
i A^{\text {Born }}=i\left[A^{\gamma, \text { Born }}+A^{Z, B o r n}\right]
$$

with

$$
\begin{gathered}
A^{\gamma, \text { Born }}=-\frac{8 \pi \alpha_{0} Q_{f}}{q^{2}} \bar{v}\left(e^{+}\right) \gamma^{\mu} p_{\mu} u\left(e^{-}\right) \\
A^{Z, \text { Born }}=\frac{4 \pi \alpha_{0}\left[I_{f}^{3}-s_{W}^{2} Q_{f}\right]}{s_{W}^{2} c_{W}^{2}\left(q^{2}-M_{Z, 0}^{2}\right)} \bar{v}\left(e^{+}\right) \gamma^{\mu} p_{\mu}\left[g_{e L, 0}^{Z} P_{L}+g_{e R, 0}^{Z} P_{R}\right] u\left(e^{-}\right)
\end{gathered}
$$

where $p$ is the outgoing sfermion momentum, $p^{\prime}$ the outgoing anti-sfermion momentum, $q=p+p^{\prime}, P_{L, R}=\left(1 \mp \gamma^{5}\right) / 2, \alpha_{0}$ is the bare QED coupling $\alpha_{0}=e_{0}^{2} / 4 \pi, s_{W}^{2}$ is the bare Salam-Weinberg angle, $g_{e L, 0}^{Z}=2 s_{W}^{2}-1, g_{e R, 0}^{Z}=2 s_{W}^{2}, I_{f}^{3}$ is the isospin third component of the final sfermion and $Q_{f}$ is the sfermion electric charge in units of $|e|$. 
It is rather convenient to introduce the chiral variables $a_{L, R}$. At the Born level, they are defined as follows:

$$
\begin{gathered}
A^{\text {Born }} \equiv \frac{8 \pi \alpha_{0}}{q^{2}} \bar{v}\left(e^{+}\right) \gamma^{\mu} p_{\mu}\left[a_{L}^{B o r n} P_{L}+a_{R}^{B o r n} P_{R}\right] u\left(e^{-}\right) \\
a_{L}^{B o r n}=-Q_{f}+\frac{\left(I_{f}^{3}-s_{W}^{2} Q_{f}\right) g_{e L, 0}^{Z}}{2 s_{W}^{2} c_{W}^{2}}=-\frac{s_{W}^{2} Q_{f}+\left(1-2 s_{W}^{2}\right) I_{f}^{3}}{2 s_{W}^{2} c_{W}^{2}} \\
a_{R}^{\text {Born }}=-Q_{f}+\frac{\left(I_{f}^{3}-s_{W}^{2} Q_{f}\right) g_{e R, 0}^{Z}}{2 s_{W}^{2} c_{W}^{2}}=\frac{I_{f}^{3}-Q_{f}}{c_{W}^{2}}
\end{gathered}
$$

We shall first treat, as we said, the processes of production of chiral sfermion-antisfermion pairs. The meaningful observables that we shall consider in this paper will be :

1) the production cross sections

$$
\sigma_{L, R}(\tilde{f})=\int_{-1}^{1} d \cos \theta\left[\frac{d \sigma_{L, R}(\tilde{f})}{d \cos \theta}\right]
$$

with

$$
\frac{d \sigma_{L, R}(\tilde{f})}{d \cos \theta}=N \frac{\pi \alpha_{0}^{2} \beta^{3}}{8 q^{2}} \sin ^{2} \theta\left|a_{L, R}(\tilde{f})\right|^{2}
$$

where $N$ is the number of colours and $\beta^{2}=1-4 m_{\tilde{f}}^{2} / q^{2}$.

At Born level it writes

$$
\sigma_{L, R}^{\text {Born }}(\tilde{f})=N_{\text {col }} \frac{\pi \alpha_{0}^{2} \beta^{3}}{6 q^{2}}\left|a_{L, R}^{B o r n}(\tilde{f})\right|^{2}
$$

Note that, at the Born level, all forward-backward asymmetries defined with

$$
\sigma_{L, R}^{F B}(\tilde{f})=\left(\int_{0}^{1}-\int_{-1}^{0}\right) d \cos \theta\left[\frac{d \sigma_{L, R}(\tilde{f})}{d \cos \theta}\right]
$$

vanish

$$
A_{F B ; L, R}^{B o r n}(\tilde{f})=\frac{\sigma_{L, R}^{F B, B o r n}(\tilde{f})}{\sigma_{L, R}^{B o r n}(\tilde{f})} \equiv 0
$$

This is a well-known feature of the coupling of a vector boson to a pair of spinless particles, which cannot generate an asymmetric $\cos \theta$ term.

At higher perturbative orders, the forward-backward asymmetries will not vanish in general. This fact will be stressed and exploited in the following part of the paper. 
2) the various longitudinal polarization asymmetries

$$
A_{L R, \tilde{f}}=\frac{\sigma_{L}(\tilde{f})-\sigma_{R}(\tilde{f})}{\sigma_{L}(\tilde{f})+\sigma_{R}(\tilde{f})}
$$

with their Born expressions

$$
A_{L R, \tilde{f}}^{\text {Born }}=\frac{\left|a_{L}^{B o r n}(\tilde{f})\right|^{2}-\left|a_{R}^{B o r n}(\tilde{f})\right|^{2}}{\left|a_{L}^{B o r n}(\tilde{f})\right|^{2}+\left|a_{R}^{B o r n}(\tilde{f})\right|^{2}}
$$

b) General one-loop treatment

In the previous Born expressions, all the involved parameters, i.e. the electric charge, the $Z$ mass and the Salam-Weinberg angle, are by definition bare ones, without unambiguously defined physical meanings. Moving to the next perturbative one-loop level, this ambiguity must be removed. In the SM case, the general procedures are well known. Briefly, the bare charge and the $Z$ mass are normally replaced by the corresponding physical quantities, defined by measurements performed at $q^{2}=0$ ("photon peak") and at $q^{2}=M_{Z}^{2}$ (" $Z$ peak"). For the bare Salam-Weinberg angle $s_{W}^{2}$, one possible convenient attitude is that of replacing it by the corresponding effective squared sine $s_{l}^{2}$, defined by measurements at the $Z$ peak, and for a thorough discussion of the related definitions we defer the reader to the existing literature [16]. With the three previous replacements and definitions, all the ultraviolet divergences at the one-loop level are automatically canceled, and the expressions of the various observables at one-loop may be written as a simple generalization of the corresponding Born quantities, by formally replacing bare parameters with physical ones, where special finite gauge-invariant combinations of one-loop diagrams enter [16].

For the processes that we want to consider in this paper, the possible approach to be followed in the MSSM is essentially similar. This can be understood without long detailed proofs if one accepts the prescriptions of the theoretical model, where by definition SUSY is broken in a "soft" way, so that no new types of ultraviolet divergences appear with respect to the SM. In particular, for the process that we are considering, the number of bare parameters that appear at Born level is still equal to three, so that the definition of three physical quantities must be sufficient to eliminate all ultraviolet divergences. Two of these quantities remain the electric charge and the $Z$ mass. The third bare parameter can still be taken as $s_{W}^{2}$, that appears both in the $Z$ coupling to the initial electron and in that to the final sfermions. For this quantity, we could always assume a redefinition that implies an extra measurement at a suitable c.m. squared energy $q_{\tilde{f}}^{2}$ and "shift" the $q_{\tilde{f}}^{2}$ dependence in a one-loop expression, where it will fix e.g. the kinematical point where to compute finite, gauge-independent combinations of self-energies, vertices and boxes to be added to a redefined "Born" term now fixed by $s_{l}^{2}\left(q_{\tilde{f}}^{2}\right)$. Alternatively, one can still perform a redefinition at the "Z peak" and start from a Born term that only contains the weak Salam-Weinberg angle measured at LEP1, SLC. The price to pay will be that in the one-loop corrections a fraction of the terms will contain contributions to be theoretically estimated at the correspondent " $\mathrm{Z}$ peak" c.m. energy where experimental information on the final $\tilde{f} \tilde{\tilde{f}}$ state does not exist. The point is that, in the asymptotic regime in which we are interested, the dependence on these 
terms will be a part of an overall constant that in the logarithmic content will disappear. Thus, in practice, the same input parameter $s_{l}^{2}\left(M_{Z}^{2}\right)$ that entered the SM case can be taken as the third theoretical input. This, we stress, will be perfectly acceptable to the extent that one is only interested in the determination of the leading logarithmic terms in a high energy expansion, which is exactly our case. With our choice, the physical expressions that will appear at one loop will contain, in their so defined "physical" Born approximation, the same expressions that were entering at the original Born level, with the bare parameters $\alpha_{0}$, $M_{0 Z}, s_{W}^{2}$ systematically replaced by $\alpha, M_{Z}, s_{l}^{2}\left(M_{Z}^{2}\right)$ and extra "corrections" generated by self-energies, vertices and boxes as depicted in Fig. (2). These will bring contributions in the asymptotic energy region that might, or might not, generate asymptotic logarithms to the various observables, in a way that we shall now illustrate.

\section{c) Asymptotic behavior of the different one-loop diagrams}

The class of diagrams that will generally contribute at one loop is shown in Fig. 2 . To be more precise, we should add the statement that, in the two diagrams (b), (c) of vertex type, also the various external self-energy insertions must be included. This will be fundamental in our approach, since the addition of those diagrams will cancel ultraviolet divergences of the "normal" vertices, making the overall contributions ultraviolet finite.

At asymptotic energies, the role of the various Figures becomes drastically different. From Fig.2a we shall obtain the known Renormalization Group (RG) linear logarithms. These will be generated from both SM and MSSM virtual pairs. Inside the SM component, there will be a gauge-dependent term due to virtual pairs of charged $W$ 's and charged would-be SM Goldstone bosons, that must be retained in a general $\xi$-gauge $(\xi \neq \infty)$ (our calculations will be systematically performed in the Feynman-t'Hooft $\xi=1$ gauge, and thus all the SM would-be Goldstone bosons contributions must be computed). This gaugedependence will be canceled, in a by now well-known way [17], by a component (the "pinch" component [18]) of the corresponding SM vertices, and we do not insist here on this fact, that has already been exhaustively discussed in previous references [3]. In our notation, the RG contribution will thus indicate the subleading (linear) logarithm generated at one loop by the sum of the self-energies and of the "pinch" components of the vertices.

In Fig.2b, the "non pinch" SM component of the initial vertices must be selected, together with the genuinely supersymmetric one of the MSSM. This operation will lead to two separate classes of contributions; the first one, coming from the vertices with SM virtual exchanges $((a b c) \equiv(\gamma e e),(Z e e),(W \nu \nu),(\nu W W))$ will generate both quadratic (DL) and linear (SL) Sudakov logarithms (note that the SM would-be Goldstone bosons and all Higgs contributions vanish due to the (assumed) vanishing electron mass). The genuine SUSY vertices, corresponding to Fig.2b with $\left((a b c) \equiv\left(\chi_{i}^{0} \tilde{e} \tilde{e}\right),\left(\chi_{i}^{+} \tilde{\nu} \tilde{\nu}\right),\left(\tilde{\nu} \chi_{i}^{+} \chi_{j}^{+}\right)\right)$(where we denote by $\chi_{i}^{0}$ and $\chi_{i}^{+}$neutralinos and charginos and we assume, again, a vanishing electron mass), will "only" generate a linear (SL) Sudakov logarithm, essentially of gauge (i.e. not of Yukawa) origin.

A similar picture is valid for the final vertices, represented in Fig.2c (SM virtual exchanges $\left((a b c) \equiv(\tilde{f} \gamma \tilde{f}),(\tilde{f} Z \tilde{f}),\left(\tilde{f}^{\prime} W \tilde{f}^{\prime}\right)\left(W \tilde{f}^{\prime} W\right)\right)$ and genuine SUSY contributions $((a b c) \equiv$ $\left.\left(\tilde{f} \chi_{i}^{0} \tilde{f}\right),\left(\tilde{f}^{\prime} \chi_{i}^{+} \tilde{f}^{\prime}\right),\left(\chi_{i}^{0} \tilde{f} \chi_{j}^{0}\right),\left(\chi_{i}^{+} \tilde{f}^{\prime} \chi_{j}^{+}\right)\right)$. Note that the diagrams with Higgs exchanges 
are now present, but vanish asymptotically and thus disappear in our analysis. This time, contributions of Yukawa type (only arising from the Higgsino component of the chargino and neutralino couplings) must be retained for third family final pairs, and we shall list them in our formulae with a proper notation. Again, SM diagrams will generate both DL and SL terms, while SUSY contributions will systematically be of SL logarithmic gauge type (and for the third family, also of Yukawa type).

Finally, there will be diagrams of box type, represented in Fig.2d. This time, a welcome simplification will appear, since only SM virtual gauge $s$-channel exchanges $((a b c d) \equiv$ $\left.(e Z \tilde{f} Z),(e \gamma \tilde{f} \gamma),(e Z \tilde{f} \gamma),(e \gamma \tilde{f} Z),\left(\nu W \tilde{f}^{\prime} W\right)\right)$ do produce asymptotic Sudakov logarithms (all other SM boxes vanish asymptotically), while all SUSY virtual box exchanges have the typical property of vanishing asymptotically. This is a simple consequence of the spin structure of the corresponding diagrams, that allows to avoid to perform several involved calculations in the final asymptotic numerical analysis. The surviving SM diagrams generate two kinds of contributions. One is of universal DL kind, when half of it is combined with a part of the final $W W$ vertex to produce a "universal" $\left(4 \ln q^{2}-\ln ^{2} q^{2}\right)$ term and the other half is combined with a part of the initial $W W$ vertex to produce a "universal" $\left(3 \ln q^{2}-\ln ^{2} q^{2}\right)$ term. The other one is of non universal kind, of SL origin and depending on $\cos \theta$, where $\theta$ is the c.m. scattering angle. All these results are essentially similar to the ones that were found in the case of SM final fermion pairs production [12, with the expected difference that a new $\simeq\left(4 \ln q^{2}-\ln ^{2} q^{2}\right)$ universal term appears, associated to the final scalar SUSY pair that is produced.

After this first qualitative discussion, that we hope to have presented in a short and understandable way, we are now ready to write the various logarithmic contributions to the considered process generated by the relevant one-loop diagrams. We shall divide them in subsets, that correspond essentially to the four components of Fig.2 (keeping in mind the previous remarks on vertex "pinch" components), trying to separate within each subset the specific constituents of different diagrams. The procedure will first list the effects on the two independent quantities $a_{L, R}$, defined by Eq. (2.4 2.6), the effects on the various observables will follow. Since the list of equations will be rather long, we shall try to eliminate, as much as possible, definitions and conventions. To render a check of our results realistically possible for the interested reader, we specify here that our SUSY Feynman rules have been taken from Rosiek's paper [19].

d) Logarithmic expansion at one loop of the scattering amplitude

At one loop, with our choice of physical inputs, we shall write the invariant scattering amplitude, for c.m. energy values $q^{2} \gg M_{W}^{2}$, in the following form:

$$
A=A^{\text {Born }}+A^{1 \text { loop }} \equiv \frac{4 \pi \alpha}{q^{2}} \bar{v}\left(e^{+}\right) \gamma^{\mu} p_{\mu}\left[a_{L} P_{L}+a_{R} P_{R}\right] u\left(e^{-}\right)
$$

with

$$
\begin{aligned}
& a_{L}=a_{L}^{\text {Born }}+a_{L}^{1 \text { loop }} \\
& a_{R}=a_{R}^{\text {Born }}+a_{R}^{1 \text { loop }}
\end{aligned}
$$


Note that we now write the Born terms as

$$
\begin{gathered}
a_{L}^{\text {Born }}=-Q_{f}+\frac{\left(I_{f}^{3}-s_{l}^{2} Q_{f}\right) g_{e L}}{2 s_{l}^{2} c_{l}^{2}}=-\frac{s_{l}^{2}\left(M_{Z}^{2}\right) Q_{f}+\left(1-2 s_{l}^{2}\right) I_{f}^{3}}{2 s_{l}^{2} c_{l}^{2}} \\
a_{R}^{\text {Born }}=-Q_{f}+\frac{\left(I_{f}^{3}-s_{l}^{2} Q_{f}\right) g_{e R}}{2 s_{l}^{2} c_{l}^{2}}=\frac{I_{f}^{3}-Q_{f}}{c_{l}^{2}}
\end{gathered}
$$

with $g_{e L}=2 s_{l}^{2}-1, g_{e R}=2 s_{l}^{2}$.

\section{Complete asymptotic 1-loop results}

We shall now write the separate contributions of RG and Sudakov kind. For the Sudakov terms, we shall divide the initial vertex contributions from the final ones and the SM virtual

effects ("gauge") from the SUSY ones. Box contributions will be, in our separation, grouped into the "final gauge" component eqs.(2.20),(2.21).

$$
\begin{aligned}
a_{L, R}^{1 \text { loop }}= & a_{L, R}^{R G}+a_{L, R}^{\text {in,gauge }}+a_{L, R}^{i n, S U S Y} \\
& +a_{L, R}^{\text {fin,gauge }}+a_{L, R}^{\text {fin,SUSY }}
\end{aligned}
$$

\section{RG terms}

$$
\begin{aligned}
a_{L}^{R G}=[ & \frac{-\alpha Q_{f}}{4 \pi}\left\{\left(\frac{32}{9} N-7\right)^{S M}+\left(3+\frac{16 N}{9}\right)^{S U S Y}\right\} \\
& +\frac{\alpha}{2 \pi s_{l}^{2} c_{l}^{2}}\left[( 2 I _ { f } ^ { 3 } + Q _ { f } ( 1 - 4 s _ { l } ^ { 2 } ) ] \left\{\frac{1}{3}\left[\frac{10-16 c_{l}^{2}}{6} N+\frac{1+42 c_{l}^{2}}{8}\right]^{S M}\right.\right. \\
& \left.-\frac{1}{4}\left[\frac{13-18 s_{l}^{2}}{6}+\left(3-8 s_{l}^{2}\right) \frac{2 N}{9}\right]^{S U S Y}\right\} \\
& +\frac{\left(I_{f}^{3}-s_{l}^{2} Q_{f}\right)\left(2 s_{l}^{2}-1\right) \alpha}{8 \pi s_{l}^{4} c_{l}^{4}}\left\{\left[\frac{20-40 c_{l}^{2}+32 c_{l}^{4}}{9} N+\frac{\left.1-2 c_{l}^{2}-42 c_{l}^{4}\right]^{S M}}{6}\right.\right. \\
& \left.\left.+\left[\frac{13-26 s_{l}^{2}+18 s_{l}^{4}}{6}+\left(3-6 s_{l}^{2}+8 s_{l}^{4}\right) \frac{2 N}{9}\right]^{S U S Y}\right\}\right] \ln \left(\frac{q^{2}}{\mu^{2}}\right) \\
a_{R}^{R G}= & {\left[\frac{-\alpha Q_{f}}{4 \pi}\left\{\left(\frac{32}{9} N-7\right)^{S M}+\left(3+\frac{16 N}{9}\right)^{S U S Y}\right\}\right.} \\
& +\frac{\alpha}{\pi s_{l}^{2} c_{l}^{2}}\left[( I _ { f } ^ { 3 } - 2 s _ { l } ^ { 2 } Q _ { f } ] \left\{\frac{1}{3}\left[\frac{10-16 c_{l}^{2}}{6} N+\frac{1+42 c_{l}^{2}}{8}\right]^{S M}\right.\right. \\
& \left.-\frac{1}{4}\left[\frac{13-18 s_{l}^{2}}{6}+\left(3-8 s_{l}^{2}\right) \frac{2 N}{9}\right]^{S U S Y}\right\} \\
& +\frac{\left(I_{f}^{3}-s_{l}^{2} Q_{f}\right) \alpha}{4 \pi s_{l}^{2} c_{l}^{4}}\left\{\left[\frac{20-40 c_{l}^{2}+32 c_{l}^{4}}{9} N+\frac{1-2 c_{l}^{2}-42 c_{l}^{4}}{6}\right]^{S M}\right. \\
& \left.\left.+\left[\frac{13-26 s_{l}^{2}+18 s_{l}^{4}}{6}+\left(3-6 s_{l}^{2}+8 s_{l}^{4}\right) \frac{2 N}{9}\right]^{S U S Y}\right\}\right] \ln \left(\frac{q^{2}}{\mu^{2}}\right)
\end{aligned}
$$


The above one-loop logarithms are of course reproduced by simply inserting in the Born expression for the cross section the running couplings $g$ and $g^{\prime}$ of $S U(2) \times U(1)$ whose scale dependence is predicted by the MSSM $\beta$ functions, see Sec.(III).

The parameter $\mu^{2}$ which appear in the previous equations will be fixed at $\mu^{2}=M_{Z}^{2}$, which is a natural and consistent choice in our approach. For further use we shall define the coefficients $c_{L, R}^{R G}$ writing:

$$
a_{L, R}^{R G} \equiv\left[a_{L, R}^{B o r n} \frac{\alpha}{\pi}\right] c_{L, R}^{R G}
$$

\section{initial gauge terms}

$$
\begin{aligned}
a_{L}^{\text {in,gauge }}= & {\left[a_{L}^{\text {Born }} \frac{\alpha}{16 \pi s_{l}^{2} c_{l}^{2}}\right]\left\{\left(1-2 s_{l}^{2}\right)^{2}\left[3 \ln \frac{q^{2}}{M_{Z}^{2}}-\ln ^{2} \frac{q^{2}}{M_{Z}^{2}}\right]\right.} \\
+ & \left.4 s_{W}^{2} c_{W}^{2}\left[3 \ln \frac{q^{2}}{M_{\gamma}^{2}}-\ln ^{2} \frac{q^{2}}{M_{\gamma}^{2}}\right]+2 c_{W}^{2}\left[3 \ln \frac{q^{2}}{M_{W}^{2}}-\ln ^{2} \frac{q^{2}}{M_{W}^{2}}\right]\right\} \\
a_{R}^{\text {in,gauge }}= & {\left[a_{R}^{\text {Born }} \frac{\alpha}{16 \pi s_{l}^{2} c_{l}^{2}}\right]\left\{4 s_{l}^{4}\left[3 \ln \frac{q^{2}}{M_{Z}^{2}}-\ln ^{2} \frac{q^{2}}{M_{Z}^{2}}\right]\right.} \\
& \left.+4 s_{l}^{2} c_{l}^{2}\left[3 \ln \frac{q^{2}}{M_{\gamma}^{2}}-\ln ^{2} \frac{q^{2}}{M_{\gamma}^{2}}\right]\right\}
\end{aligned}
$$

In the above equation, the mass $M_{\gamma}$ refers to the cut-off which separates in the photon exchange contribution the ultraviolet from the infrared part. Since the main purpose of this paper is the determination of asymptotic electroweak effects (neglecting in particular soft photon emission effects which are determined by QED only if the experimental cut $\Delta E$ is smaller then $M_{Z} \simeq M_{W}$, we shall set from now on $M_{\gamma}=M_{Z}=M_{W}$. With this choice we can check that for both the left and right terms we can write

$$
a_{L, R}^{\text {in,gauge }} \equiv\left[a_{L, R}^{\text {Born }} \frac{\alpha}{\pi}\right] c_{L, R}^{\text {in,gauge }}
$$

with

$$
c_{L, R}^{\text {in,gauge }}=\frac{1}{4}\left(\frac{I_{e}\left(I_{e}+1\right)}{s_{l}^{2}}+\frac{Y_{e}^{2}}{4 c_{l}^{2}}\right)\left[3 \ln \frac{q^{2}}{M_{W}^{2}}-\ln ^{2} \frac{q^{2}}{M_{W}^{2}}\right]
$$

where $Y_{e}=2\left(Q_{e}-I_{e}^{3}\right)$ and the quantum numbers required in the calculation of $a_{L}\left(a_{R}\right)$ are those of left (right) electrons. 


\section{initial SUSY terms}

In the following equations we have introduced different SUSY scales $M_{c h}, M_{\text {neut }}$ for the chargino and neutralino contributions,

$$
\begin{gathered}
a_{L}^{i n, S U S Y}=\left[a_{L}^{\text {Born }} \frac{-\alpha}{16 \pi s_{l}^{2} c_{l}^{2}}\right]\left\{\left[\ln \frac{q^{2}}{M_{\text {neut }}^{2}}\right]+2 c_{l}^{2}\left[\ln \frac{q^{2}}{M_{c h}^{2}}\right]\right\} \\
a_{R}^{i n, S U S Y}=\left[a_{R}^{\text {Born }} \frac{-\alpha}{16 \pi s_{l}^{2} c_{l}^{2}}\right] 4 s_{l}^{2}\left[\ln \frac{q^{2}}{M_{\text {neut }}^{2}}\right]
\end{gathered}
$$

but in practice we will take them equal to a common SUSY scale $M_{S U S Y}$ and write

$$
a_{L, R}^{i n, S U S Y} \equiv\left[a_{L, R}^{\text {Born }} \frac{\alpha}{\pi}\right] c_{L, R}^{i n, S U S Y}
$$

with

$$
c_{L, R}^{i n, S U S Y}=-\frac{1}{4}\left(\frac{I_{e}\left(I_{e}+1\right)}{s_{W}^{2}}+\frac{Y_{e}^{2}}{4 c_{l}^{2}}\right)\left[\ln \frac{q^{2}}{M_{S U S Y}^{2}}\right]
$$

\section{final gauge terms}

$$
\begin{aligned}
& a_{L}^{f i n, g a u g e}=\frac{-\alpha}{8 \pi s_{l}^{4} c_{l}^{4}}\left\{[ Q _ { f } s _ { l } ^ { 2 } + I _ { f } ^ { 3 } ( 1 - 2 s _ { W } ^ { 2 } ) ] \left(\left(I_{f}^{3}-s_{l}^{2} Q_{f}\right)^{2}\left[4 \ln \frac{q^{2}}{M_{Z}^{2}}-\ln ^{2} \frac{q^{2}}{M_{Z}^{2}}\right]\right.\right. \\
& \left.+s_{l}^{2} c_{l}^{2} Q_{f}^{2}\left[4 \ln \frac{q^{2}}{M_{\gamma}^{2}}-\ln ^{2} \frac{q^{2}}{M_{\gamma}^{2}}\right]\right) \\
& \left.+\left(\left[Q_{f} \frac{s_{l}^{2} c_{l}^{2}}{2}-\left(2 I_{f}^{3}\right) \frac{c_{l}^{2}}{4}\right]\left[4 \ln \frac{q^{2}}{M_{W}^{2}}-\ln ^{2} \frac{q^{2}}{M_{W}^{2}}\right]\right)_{\tilde{f}_{L} \text { only }}\right\} \\
& -\left(\frac{\alpha}{16 \pi s_{l}^{4}}\left(2 I_{f}^{3}\right)\left[4 \ln \frac{q^{2}}{M_{W}^{2}}-\ln ^{2} \frac{q^{2}}{M_{W}^{2}}\right]-\frac{\alpha}{4 \pi s_{l}^{4}}\left(2 I_{f}^{3}\right) \ln \frac{q^{2}}{M_{W}^{2}} \ln \frac{1+\left(2 I_{f}^{3}\right) \cos \theta}{2}\right)_{\tilde{f}_{L} \text { only }} \\
& -\frac{\alpha}{4 \pi s_{l}^{4} c_{l}^{4}}\left(I_{f}^{3}-s_{l}^{2} Q_{f}\right)^{2}\left(2 s_{l}^{2}-1\right)^{2} \ln \frac{q^{2}}{M_{Z}^{2}} \ln \frac{1-\cos \theta}{1+\cos \theta} \\
& -\frac{\alpha}{\pi} Q_{f}^{2} \ln \frac{q^{2}}{M_{g}^{2}} \ln \frac{1-\cos \theta}{1+\cos \theta} \\
& +\frac{\alpha}{\pi s_{l}^{2} c_{l}^{2}} Q_{f}\left(I_{f}^{3}-s_{W}^{2} q_{f}\right)\left(2 s_{l}^{2}-1\right) \ln \frac{q^{2}}{M_{\gamma Z}^{2}} \ln \frac{1-\cos \theta}{1+\cos \theta} \\
& a_{R}^{\text {fin,gauge }}=\frac{-\alpha}{4 \pi s_{l}^{2} c_{l}^{4}}\left[Q_{f}-I_{f}^{3}\right]\left\{\left(I_{f}^{3}-s_{l}^{2} Q_{f}\right)^{2}\left[4 \ln \frac{q^{2}}{M_{Z}^{2}}-\ln ^{2} \frac{q^{2}}{M_{Z}^{2}}\right]\right. \\
& \left.+s_{l}^{2} c_{l}^{2} Q_{f}^{2}\left[4 \ln \frac{q^{2}}{M_{\gamma}^{2}}-\ln ^{2} \frac{q^{2}}{M_{\gamma}^{2}}\right]+\left(\frac{c_{l}^{2}}{2}\left[4 \ln \frac{q^{2}}{M_{W}^{2}}-\ln ^{2} \frac{q^{2}}{M_{W}^{2}}\right]\right)_{\tilde{f}_{L} \text { only }}\right\} \\
& -\frac{\alpha}{\pi c_{W}^{4}}\left(I_{f}^{3}-s_{l}^{2} Q_{f}\right)^{2} \ln \frac{q^{2}}{M_{Z}^{2}} \ln \frac{1-\cos \theta}{1+\cos \theta} \\
& -\frac{\alpha}{\pi} Q_{f}^{2} \ln \frac{q^{2}}{M_{g}^{2}} \ln \frac{1-\cos \theta}{1+\cos \theta} \\
& +\frac{2 \alpha}{\pi c_{l}^{2}} Q_{f}\left(I_{f}^{3}-s_{l}^{2} Q_{f}\right) \ln \frac{q^{2}}{M_{\gamma Z}^{2}} \ln \frac{1-\cos \theta}{1+\cos \theta}
\end{aligned}
$$


Identifying all gauge mass scales with $M_{W}$ as we did for the initial gauge terms, one can factorize the Born amplitudes and write

$$
\begin{aligned}
& a_{L, R}^{\text {fin,gauge }} \equiv\left[a_{L, R}^{\text {Born }} \frac{\alpha}{\pi}\right] c_{L, R}^{\text {fin,gauge }} \\
& c_{L}^{\text {fin,gauge }}\left(\tilde{f}_{L}\right)=\frac{1}{4}\left(\frac{I_{f}\left(I_{f}+1\right)}{s_{l}^{2}}+\frac{Y_{f}^{2}}{4 c_{l}^{2}}\right)\left(4 \ln \frac{q^{2}}{M_{W}^{2}}-\ln ^{2} \frac{q^{2}}{M_{W}^{2}}\right)+ \\
& +\left(\frac{I_{f}^{3}}{2 s_{l}^{2}}+\frac{Y_{f}}{4 c_{l}^{2}}\right)\left[\ln \frac{q^{2}}{M^{2}}\right] \ln \frac{1-\cos \theta}{1+\cos \theta}+ \\
& +\frac{1}{4 a_{L}^{B o r n}\left(\widetilde{f}_{L}\right) s_{l}^{4}}\left(2 I_{f}^{3}\right)\left[\ln \frac{q^{2}}{M^{2}}\right] \ln \frac{1+2 I_{f}^{3} \cos \theta}{2} \\
& c_{L}^{\text {fin,gauge }}\left(\widetilde{f}_{R}\right)=\frac{Y_{f}^{2}}{16 c_{l}^{2}}\left(4 \ln \frac{q^{2}}{M_{W}^{2}}-\ln ^{2} \frac{q^{2}}{M_{W}^{2}}\right)+ \\
& +\frac{Y_{f}}{2 c_{l}^{2}}\left[\ln \frac{q^{2}}{M^{2}}\right] \ln \frac{1-\cos \theta}{1+\cos \theta} \\
& c_{R}^{\text {fin,gauge }}\left(\widetilde{f}_{L}\right)=\frac{1}{4}\left(\frac{I_{f}\left(I_{f}+1\right)}{s_{l}^{2}}+\frac{Y_{f}^{2}}{4 c_{l}^{2}}\right)\left(4 \ln \frac{q^{2}}{M_{W}^{2}}-\ln ^{2} \frac{q^{2}}{M_{W}^{2}}\right)+ \\
& +\frac{Y_{f}}{2 c_{l}^{2}}\left[\ln \frac{q^{2}}{M^{2}}\right] \ln \frac{1-\cos \theta}{1+\cos \theta} \\
& c_{R}^{\text {fin,gauge }}\left(\widetilde{f}_{R}\right)=\frac{Y_{f}^{2}}{16 c_{l}^{2}}\left(4 \ln \frac{q^{2}}{M_{W}^{2}}-\ln ^{2} \frac{q^{2}}{M_{W}^{2}}\right)+ \\
& +\frac{Y_{f}}{2 c_{l}^{2}}\left[\ln \frac{q^{2}}{M^{2}}\right] \ln \frac{1-\cos \theta}{1+\cos \theta}
\end{aligned}
$$




\section{final SUSY terms}

They are also written in the form

$$
a_{L, R}^{f i n, S U S Y} \equiv\left[a_{L, R}^{\text {Born }} \frac{\alpha}{\pi}\right] c_{L, R}^{f i n, S U S Y}
$$

with

$$
\begin{aligned}
& c_{L}^{f i n, S U S Y}\left(\tilde{u}_{L}, \tilde{d}_{L}\right)=c_{R}^{f i n, S U S Y}\left(\tilde{u}_{L}, \tilde{d}_{L}\right) \\
& =\left\{-\frac{1}{2}\left(\frac{I_{f}\left(I_{f}+1\right)}{s_{l}^{2}}+\frac{Y_{f}^{2}}{4 c_{l}^{2}}\right)-\frac{1}{8 M_{W}^{2} s_{l}^{2}}\left[\frac{m_{u}^{2}}{\sin ^{2} \beta}+\frac{m_{d}^{2}}{\cos ^{2} \beta}\right]\right\}\left[\ln \frac{q^{2}}{M_{S U S Y}^{2}}\right] \\
& c_{L}^{f i n, S U S Y}\left(\tilde{u}_{R}\right)=c_{R}^{f i n, S U S Y}\left(\tilde{u}_{R}\right) \\
& =\left\{-\frac{1}{2}\left(\frac{I_{f}\left(I_{f}+1\right)}{s_{l}^{2}}+\frac{Y_{f}^{2}}{4 c_{l}^{2}}\right)-\frac{1}{4 M_{W}^{2} s_{l}^{2}} \frac{m_{u}^{2}}{\sin ^{2} \beta}\right\}\left[\ln \frac{q^{2}}{M_{S U S Y}^{2}}\right] \\
& c_{L}^{f i n, S U S Y}\left(\tilde{d}_{R}\right)=c_{R}^{f i n, S U S Y}\left(\tilde{d}_{R}\right) \\
& =\left\{-\frac{1}{2}\left(\frac{I_{f}\left(I_{f}+1\right)}{s_{l}^{2}}+\frac{Y_{f}^{2}}{4 c_{l}^{2}}\right)-\frac{1}{4 M_{W}^{2} s_{l}^{2}} \frac{m_{d}^{2}}{\cos ^{2} \beta}\right\}\left[\ln \frac{q^{2}}{M_{S U S Y}^{2}}\right] \\
& c_{L}^{f i n, S U S Y}\left(\tilde{l}_{L}\right)=c_{R}^{f i n, S U S Y}\left(\tilde{l}_{L}\right)=-\frac{1}{2}\left(\frac{I_{f}\left(I_{f}+1\right)}{s_{l}^{2}}+\frac{Y_{f}^{2}}{4 c_{l}^{2}}\right)\left[\ln \frac{q^{2}}{M_{S U S Y}^{2}}\right] \\
& c_{L}^{\text {fin }, S U S Y}\left(\tilde{l}_{R}\right)=c_{R}^{f i n, S U S Y}\left(\tilde{l}_{R}\right)=-\frac{1}{2}\left(\frac{I_{f}\left(I_{f}+1\right)}{s_{l}^{2}}+\frac{Y_{f}^{2}}{4 c_{l}^{2}}\right)\left[\ln \frac{q^{2}}{M_{S U S Y}^{2}}\right]
\end{aligned}
$$

An important feature of our approach is that, in the asymptotic regime, due to the unitarity properties of the chargino and neutralino mixing matrices, the only remaining parameters are $\tan \beta$ and the SUSY scale which appears in the logarithmic terms. The role of $\tan \beta$ will be discussed in detail in Sect.V . 
e) Extension of the results to the case of charged or neutral Higgses

All the results written above for $\tilde{\bar{f}}_{L, R} \tilde{f}_{L, R}$ directly apply to the case of charged Higgses $H^{+} H^{-}$or of neutral Higgses $H_{a}^{0} H_{b+2}^{0}$, where the labels $(a=1,2, b=1,2)$ refer to

$$
H_{1}^{0}=H^{0}, \quad H_{2}^{0}=h^{0}, \quad H_{3}^{0}=A^{0}, \quad H_{4}^{0}=\varphi^{0}
$$

( $\varphi^{0}$ is the neutral Goldstone boson), defining $p$ as the outgoing $H^{-}$or $H_{a}^{0}$ momentum and $p^{\prime}$ the outgoing $H^{+}$or $H_{b+2}^{0}$ momentum, and

- for $H^{+} H^{-} \quad Q_{f}=-1 \quad I_{f}^{3}=-\frac{1}{2} \quad Y_{f}=-1$

- for $H^{0} H^{0^{\prime}} \quad Q_{f}=0 \quad I_{f}^{3}=\frac{1}{2} \quad Y_{f}=-1$

In the case of charged Higgs bosons, the Born terms are

$$
a_{L}^{B o r n}(H)=\frac{1}{4 s_{l}^{2} c_{l}^{2}} \quad a_{R}^{B o r n}(H)=\frac{1}{2 c_{l}^{2}}
$$

so that all initial gauge, initial SUSY, final gauge one loop terms can be taken from the sfermion case with the appropriate values of $I_{f}^{3}$ and $Y_{f}$ given above. The final SUSY and heavy fermion terms are :

$$
a_{L, R}^{f i n, S U S Y} \equiv\left[a_{L, R}^{\text {Born }} \frac{\alpha}{\pi}\right] c_{L, R}^{f i n, S U S Y}
$$

with

$$
\begin{aligned}
c_{L}^{f i n, S U S Y}(H) & =c_{R}^{f i n, S U S Y}(H)=\left\{-\frac{1}{2}\left(\frac{I_{H}\left(I_{H}+1\right)}{s_{l}^{2}}+\frac{Y_{H}^{2}}{4 c_{l}^{2}}\right)\right. \\
& \left.-\frac{3}{8 s_{l}^{2} M_{W}^{2}}\left(m_{d}^{2} \tan ^{2} \beta+m_{u}^{2} \cot ^{2} \beta\right)\right\}\left[\ln \frac{q^{2}}{M_{S U S Y}^{2}}\right]
\end{aligned}
$$

(note the color factor 3 arising from the quark triangle loop, which will enhance these contributions as compared to the case of final squarks).

In the case of neutral Higgs bosons the Born $\gamma$ exchange is missing and the $Z$ exchange is multiplied (with respect to the previous cases) by $i A_{a b}$ where

$$
A_{a b}=\left(\begin{array}{cc}
-\sin (\alpha-\beta) & -\cos (\alpha-\beta) \\
-\cos (\alpha-\beta) & \sin (\alpha-\beta)
\end{array}\right)
$$

The specific cases of use in this paper are

$$
\begin{aligned}
e^{+} e^{-} \rightarrow H A: A_{a b} & =-\sin (\alpha-\beta) \\
e^{+} e^{-} \rightarrow h A: A_{a b} & =-\cos (\alpha-\beta)
\end{aligned}
$$

with the corresponding Born terms

$$
a_{a b}^{L, B o r n}=\frac{1-2 s_{l}^{2}}{4 s_{l}^{2} c_{l}^{2}} A_{a b} \quad a_{a b}^{R, B o r n}=-\frac{1}{2 c_{l}^{2}} A_{a b}
$$


The initial gauge and initial SUSY one loop terms can also be taken from the sfermion case with the appropriate values of $I_{f}^{3}$ and $Y_{f}$ given above. The final gauge terms are

$$
a_{L, R ;(a b)}^{\text {fin,gauge }} \equiv\left[a_{(a b)}^{L, R ; B \text { Born }} \frac{\alpha}{\pi}\right] c_{L, R}^{\text {fin,gauge }}(a b)
$$

with

$$
\begin{aligned}
c_{L}^{\text {fin,gauge }}(a b) & =\frac{1}{4}\left(\frac{I_{f}\left(I_{f}+1\right)}{s_{l}^{2}}+\frac{Y_{f}^{2}}{4 c_{l}^{2}}\right)\left(4 \ln \frac{q^{2}}{M_{W}^{2}}-\ln ^{2} \frac{q^{2}}{M_{W}^{2}}\right) \\
& -\frac{1}{2 a_{L ;(a b)}^{\text {Born }}\left(\widetilde{f}_{L}\right) s_{l}^{4}}\left(2 I_{f}^{3}\right)\left[\ln \frac{q^{2}}{M^{2}}\right]\left[\ln \frac{1-\cos \theta}{2}+\ln \frac{1+\cos \theta}{2}\right] \\
c_{R}^{\text {fin,gauge }}(a b) & =\frac{1}{4}\left(\frac{I_{f}\left(I_{f}+1\right)}{s_{l}^{2}}+\frac{Y_{f}^{2}}{4 c_{l}^{2}}\right)\left(4 \ln \frac{q^{2}}{M_{W}^{2}}-\ln ^{2} \frac{q^{2}}{M_{W}^{2}}\right)
\end{aligned}
$$

and the final SUSY and heavy fermion terms can also be written as

$$
a_{L, R ;(a b)}^{f i n, S U S Y} \equiv\left[a_{(a b)}^{L, R ; B o r n} \frac{\alpha}{\pi}\right] c_{L, R}^{f i n, S U S Y}(a b)
$$

with

$$
\begin{aligned}
c_{L}^{f i n, S U S Y}(a b) & =c_{R}^{f i n, S U S Y}(a b)=\left\{-\frac{1}{2}\left(\frac{I_{H}\left(I_{H}+1\right)}{s_{l}^{2}}+\frac{Y_{H}^{2}}{4 c_{l}^{2}}\right)\right. \\
& \left.-\frac{3}{8 s_{l}^{2} M_{W}^{2}} f(a b)\right\}\left[\ln \frac{q^{2}}{M_{S U S Y}^{2}}\right]
\end{aligned}
$$

and

$$
\begin{gathered}
f(H A)=\frac{1}{\sin (\beta-\alpha)}\left[\frac{\sin \alpha \cos \beta}{\sin ^{2} \beta} m_{u}^{2}-\frac{\cos \alpha \sin \beta}{\cos ^{2} \beta} m_{d}^{2}\right] \\
f(h A)=-\frac{1}{\cos (\beta-\alpha)}\left[\frac{\cos \alpha \cos \beta}{\sin ^{2} \beta} m_{u}^{2}+\frac{\sin \alpha \sin \beta}{\cos ^{2} \beta} m_{d}^{2}\right]
\end{gathered}
$$

As one can observe, in the neutral Higgs case there appears one more SUSY parameter $(\alpha)$ than in all other cases, which would require a separate analysis that we feel is beyond the purposes of this first paper. We shall treat the neutral Higgs production in more general SUSY models (also beyond the MSSM) in a dedicated forthcoming paper. 


\section{f) Logarithmic expansion of the observable quantities}

The logarithmic expansion of the various observables can be now straightforwardly de-

rived from the eqs.(2.22), (2.26) (2.30), (2.33), (2.38), (2.47), (2.53), (2.54, (2.56). At one loop, we obtain the following expressions for the polarized angular distributions:

$$
\frac{d \sigma_{L, R ; f}}{d \cos \theta}=N_{c o l} \frac{\pi \alpha^{2} \beta^{3}}{8 q^{2}} \sin ^{2} \theta\left|a_{L, R ; f}\right|^{2}
$$

with the first order expansion

$$
\left|a_{L, R ; f}\right|^{2}=\left|a_{L, R ; f}^{B o r n}\right|^{2}\left[1+2 \delta_{L, R}(f)\right]
$$

where

$$
\begin{aligned}
\delta_{L, R}(f)= & {\left[\frac{\alpha}{\pi}\right]\left\{c_{L, R}^{R G}(f)+c_{L, R}^{\text {in gauge }}(f)+c_{L, R}^{f \text { in gauge }}(f)\right.} \\
& \left.+c_{L, R}^{\text {in } S U S Y}(f)+c_{L, R}^{f \text { in } S U S Y}(f)\right\}
\end{aligned}
$$

and the various $c_{L, R}^{i}(f)$ can be read off the corresponding previous eqs.(2.22), (2.26) (2.30), (2.33), (2.38), (2.47),(2.53), (2.54, (2.56).

We can then consider the integrated observables. With this purpose, we define the Born quantitiest"

$$
\begin{aligned}
& \rho_{L, f}=\frac{\left|a_{L}^{\text {Born }}\right|^{2}}{\left|a_{L}^{\text {Born }}\right|^{2}+\left|a_{R}^{\text {Born }}\right|^{2}}=\frac{\left[Q_{f} s_{l}^{2}+I_{3, f}\left(1-2 s_{l}^{2}\right)\right]^{2}}{\left[Q_{f} s_{l}^{2}+I_{3, f}\left(1-2 s_{l}^{2}\right)\right]^{2}+4 s_{l}^{4}\left(I_{3, f}-Q_{f}\right)^{2}} \\
& \rho_{R, f}=\frac{\left|a_{R}^{\text {Born }}\right|^{2}}{\left|a_{L}^{\text {Born }}\right|^{2}+\left|a_{R}^{\text {Born }}\right|^{2}}=\frac{4 s_{l}^{4}\left(I_{3, f}-Q_{f}\right)^{2}}{\left[Q_{f} s_{l}^{2}+I_{3, f}\left(1-2 s_{l}^{2}\right)\right]^{2}+4 s_{l}^{4}\left(I_{3, f}-Q_{f}\right)^{2}}
\end{aligned}
$$

and the integration of the relative 1 loop effects with respect to the scattering angle (note that there are angular dependent parts in the $\delta_{L, R}(f)$ due to box contributions)

$$
\begin{gathered}
N_{L, R ; f}^{\mathrm{full}}=\int_{-1}^{1} d \cos \theta \sin ^{2} \theta \quad\left[\delta_{L, R}(f)\right] \\
N_{L, R ; f}^{\mathrm{FB}}=\left(\int_{0}^{1}-\int_{-1}^{0}\right) d \cos \theta \sin ^{2} \theta \quad\left[\delta_{L, R}(f)\right]
\end{gathered}
$$

For real $a_{L, R}$ the relative effect in the total cross section and the absolute shifts in the asymmetries are

$$
\frac{\delta \sigma}{\sigma}=2 \frac{3}{4}\left[\rho_{L, f} N_{L, f}^{\text {full }}+\rho_{R, f} N_{R, f}^{\text {full }}\right]
$$

\footnotetext{
${ }^{1}$ Notice that $\rho_{L, f_{R}}=1 / 5$ and $\rho_{R, f_{R}}=4 / 5$.
} 


$$
\begin{gathered}
\delta A_{L R, f}=4 \rho_{L, f} \rho_{R, f} \frac{3}{4}\left(N_{L, f}^{\mathrm{full}}-N_{R, f}^{\mathrm{full}}\right) \\
A_{F B, f} \equiv \delta A_{F B, f}=\frac{3}{2}\left(\rho_{L, f} N_{L, f}^{F B}+\rho_{R f} N_{R f}^{F B}\right)
\end{gathered}
$$

We stress that there is no forward-backward asymmetry at Born level for a pair of scalar particles. The contribution from angular independent terms cancel, thus, this asymmetry arises at 1-loop order and is only due to the angular dependent box terms appearing in the above contributions called "final gauge". The $W$ box only contributes final scalar doublets ( $\tilde{f}_{L}$ or charged, neutral Higgses) labelled with " $\tilde{f}_{L}$ only". The $\gamma+Z$ contribute all final states. A simple analytical expression results:

$$
\begin{gathered}
\frac{3}{2} N_{L, f}^{F B}=-\frac{\alpha}{\pi}(1-4 \ln 2)\left\{\left[\frac{1}{8 s_{l}^{4}} \frac{1}{a_{L, f}^{B o r n}} \ln \frac{q^{2}}{M_{W}^{2}}\right]_{\tilde{f}_{L} \text { only }}+a_{L, f}^{B \text { orn }} \ln \frac{q^{2}}{M_{Z}^{2}}\right\} \\
\frac{3}{2} N_{R, f}^{F B}=-\frac{\alpha}{\pi}(1-4 \ln 2) a_{R, f}^{B o r n} \ln \frac{q^{2}}{M_{Z}^{2}}
\end{gathered}
$$

where we used $M_{\gamma}=M_{\gamma Z}=M_{Z}$ in the $\gamma+Z$ boxes.

Now we conclude this (unavoidly) long Sect.II, where the complete DL and SL terms have been computed at the one-loop level. In the forthcoming Sect.III we shall perform a resummation of all the logarithmic terms to subleading logarithmic accuracy in a convenient asymptotic (Sudakov) regime, and show that the calculation does coincide at the one-loop level with that of Sect.II. 


\section{RESUMMATION OF SUBLEADING SUDAKOV LOGARITHMS IN THE MSSM}

In the previous section we have calculated one loop Sudakov logarithms in scalar production at a linear $e^{+} e^{-}$collider at large energies in the MSSM.

With the expected experimental precision in the one percent regime at such a machine, the need for a theoretical treatment to the same accuracy was already discussed in the introduction. Recently, the treatment of electroweak Sudakov logarithms in the SM revealed the fact that for that purpose at least a two loop treatment to SL accuracy is indicated for energies above $\mathrm{TeV}$ energies [4]. For SM processes a general method of obtaining DL corrections to all orders was presented in Ref. [4] (Fadin et al.) in the context of the infrared evolution equation method. If we assume that the mass scale of the sfermions or Higgs particles are not much larger than the weak scale, these results can be applied straightforwardly to the MSSM since the gauge couplings are preserved under supersymmetry and no additional spin 1 particles are exchanged.

In case the superpartner masses are larger than $500 \mathrm{GeV}$, additional double logarithms need to be taken into account in a way outlined in Ref. [4 (Fadin et al.). In the following we assume that we can neglect such terms, i.e. that all MSSM scalars have a mass below $500 \mathrm{GeV}$.

At the subleading level, the situation in general is less clear at higher orders. For SL angular dependent terms, the same reasoning as above goes through since they originate only from the exchange of spin 1 gauge bosons and can thus be resummed as in the SM [5]. Box-type diagrams exchanging supersymmetric particles in the s-channel do not contribute to SL angular terms. The same holds for all universal SL corrections which involve the exchange of SM particles [6] since they are properties of the external particles only.

New types of SL Sudakov corrections are, however, involved in the exchange of supersymmetric particles as discussed in the previous sections at the one loop level. We begin with the corrections contributing in particular the Yukawa terms from the final state corrections. In order to see how these corrections enter into two loop SL calculations, we need to consider the diagrams displayed in Fig. 3. The corresponding two loop amplitudes read

$\int \frac{d^{n} l}{(4 \pi)^{n}} \int \frac{d^{n} k}{(4 \pi)^{n}} \frac{\left(p_{1}-p_{2}\right)_{\nu} \operatorname{Tr}\left[\left(G_{r} \omega_{r}+G_{l} \omega_{l}\right)\left(\not k-\not p_{1}\right) 2 \not p_{2}\left(\not k-\not p_{1}+l\right)\left(G_{r} \omega_{r}+G_{l} \omega_{l}\right) \not k\right]}{\left(l^{2}-\lambda^{2}\right)\left(p_{2}+l\right)^{2}\left(p_{1}-l\right)^{2} k^{2}\left(k-p_{1}\right)^{2}\left(k-p_{1}+l\right)^{2}}$

$\int \frac{d^{n} l}{(4 \pi)^{n}} \int \frac{d^{n} k}{(4 \pi)^{n}} \frac{\left(p_{1}-p_{2}\right)_{\nu} \operatorname{Tr}\left[\left(G_{r} \omega_{r}+G_{l} \omega_{l}\right)\left(\not k-\not p_{1}+l\right)\left(G_{r} \omega_{r}+G_{l} \omega_{l}\right) \not k\right] 4 p_{1} p_{2}}{\left(l^{2}-\lambda^{2}\right)\left(p_{2}+l\right)^{2}\left(p_{1}-l\right)^{2} k^{2}\left(k-p_{1}+l\right)^{2}\left(p_{1}-l\right)^{2}}$

where we omit common factors and the scalar masses assuming $m_{s} \sim \lambda$ for clarity. The $G_{r, l}$ denote the chiral Yukawa couplings and $\omega_{r, l}=\frac{1}{2}\left(1 \pm \gamma_{5}\right)$. The gauge coupling is written in the symmetric basis for clarity since we are considering a regime where $q^{2}=\left(p_{1}-p_{2}\right)^{2} \gg M^{2}$, where $M$ is the gauge boson mass. In any case, local gauge invariance is not violated in the SM and for heavy particles in the high energy limit, we can perform the calculation in a basis which is more convenient. For our purposes we need to investigate terms containing three large logarithms in those diagrams. Since the fermion loops at one loop only yield a single logarithm it is clear that the gauge boson loop momentum $l$ must be soft. Thus we need to show that the UV logarithm originating from the $k$ integration is identical (up 
to the sign) in both diagrams. We can therefore neglect the loop momentum $l$ inside the fermion loop. We find for the fermion loop vertex $\Lambda^{\mu}\left(p_{1}^{2}, 0, p_{1}^{2}\right)$ belonging to Eq. (3.1):

$$
\begin{aligned}
& \frac{\operatorname{Tr}\left[\left(G_{r} \omega_{r}+G_{l} \omega_{l}\right)\left(\not k-\not p_{1}\right) \gamma^{\mu}\left(\not k-\not p_{1}\right)\left(G_{r} \omega_{r}+G_{l} \omega_{l}\right) \not k\right]}{k^{2}\left(k-p_{1}\right)^{2}\left(k-p_{1}\right)^{2}} \\
= & \frac{4 G_{r} G_{l}\left(2 p_{1}^{\mu}\left(k^{2}-p_{1} k\right)+k^{\mu}\left(p_{1}^{2}-k^{2}\right)\right)}{k^{2}\left(k-p_{1}\right)^{4}}
\end{aligned}
$$

This we need to compare with the self energy loop $\Sigma\left(p_{1}^{2}\right)$ from Eq. (3.2):

$$
\begin{aligned}
& \frac{\partial}{\partial p_{1 \mu}} \frac{\operatorname{Tr}\left[\left(G_{r} \omega_{r}+G_{l} \omega_{l}\right)\left(\not k-\not p_{1}\right)\left(G_{r} \omega_{r}+G_{l} \omega_{l}\right) \not k\right]}{k^{2}\left(k-p_{1}\right)^{2}} \\
= & \frac{\partial}{\partial p_{1_{\mu}}} \frac{4 G_{r} G_{l}\left(p_{1} k-k^{2}\right)}{k^{2}\left(k-p_{1}\right)^{2}}=4 G_{r} G_{l} \frac{2 p_{1}^{\mu}\left(k^{2}-p_{1} k\right)+k^{\mu}\left(p_{1}^{2}-k^{2}\right)}{k^{2}\left(k-p_{1}\right)^{4}}
\end{aligned}
$$

In short we can write

$$
\frac{\partial}{\partial p_{1_{\mu}}} \Sigma\left(p_{1}^{2}\right)=\Lambda^{\mu}\left(p_{1}^{2}, 0, p_{1}^{2}\right)
$$

Thus, we have established a Ward identity for arbitrary Yukawa couplings of scalars to fermions and thus, the identity of the UV singular contributions. The relative sign is such that the generated SL logarithms of the diagrams in Fig. 3 cancel each other. The existence of such an identity is not surprising since it expresses the fact that also the Yukawa sector is gauge invariant since supersymmetry preserves the gauge symmetry. in perturbation theory to SL accuracy. For the same reason the SM-Yukawa terms were found to exponentiate in Refs. [6]. Also in an axial gauge the corrections can be seen to factorize accordingly since in this gauge DL terms originate only from on-shell two point functions.

We are thus left with gauge boson corrections to the original vertices in the on-shell renormalization scheme such as depicted in Fig. 1 . At high energies we can therefore employ the non-Abelian version of Gribov's bremsstrahlung theorem in accordance with the SM case [1] (Fadin et al.).

Analogously, it is easy to see that also the diagrams depicted in Fig. 5 form the initial state exchange of supersymmetric scalar particles lead to Ward identities. For the respective vertex and self energy contributions we have verified that the corresponding Eq. (3.5) is fulfilled. Thus the same reasoning as above can be applied and the exponentiation at the SL level is established.

For our purposes here we omit the soft photon regime for now (which is determined by QED only if we impose an experimental energy resolution below the weak scale [6]) and focus only on the novel higher order Sudakov corrections in the MSSM. For clarity and later convenience, we use a common mass scale in all logarithms below. This is not fully correct to SL accuracy in the DL terms as discussed below but can easily be rectified using the scales found in the one loop calculation presented in the previous sections. In the high energy regime one then has the following result for sfermion production to SL-accuracy relative to the Born cross section (with $t=-\frac{q^{2}}{2}(1-\cos \theta), \quad u=-\frac{q^{2}}{2}(1+\cos \theta)$ ):

\footnotetext{
${ }^{2}$ We denote the chirality $\mathrm{L}, \mathrm{R}$ by the the index $\alpha$ with $-L=R$.
} 


$$
\begin{aligned}
& d \sigma_{e_{-\alpha}^{+} e_{\alpha}^{-} \longrightarrow \overline{\tilde{f}}_{-\beta} \tilde{f}_{\beta}}^{\mathrm{SL}}=d \sigma_{e_{-\alpha}^{+} e_{\alpha}^{-} \longrightarrow \overline{\tilde{f}}_{-\beta} \tilde{f}_{\beta}}^{\text {Born }} \times \\
& \exp \left\{-\frac{g^{2}\left(m_{\tilde{f}}^{2}\right)}{4 \pi^{2}} I_{e_{\alpha}^{-}}\left(I_{e_{\alpha}^{-}}+1\right)\left[\frac{1}{c} \ln \frac{q^{2}}{m_{\tilde{f}}^{2}}\left(\ln \frac{g^{2}\left(m_{\tilde{f}}^{2}\right)}{g^{2}\left(q^{2}\right)}-1\right)+\frac{1}{c^{2}} \ln \frac{g^{2}\left(m_{\tilde{f}}^{2}\right)}{g^{2}\left(q^{2}\right)}\right]\right. \\
& -\frac{g^{\prime 2}\left(m_{\tilde{f}}^{2}\right) Y_{e_{\alpha}^{-}}^{2}}{16 \pi^{2}}\left[\frac{1}{c^{\prime}} \ln \frac{q^{2}}{m_{\tilde{f}}^{2}}\left(\ln \frac{g^{\prime 2}\left(m_{\tilde{f}}^{2}\right)}{g^{\prime 2}\left(q^{2}\right)}-1\right)+\frac{1}{c^{\prime 2}} \ln \frac{g^{\prime 2}\left(m_{\tilde{f}}^{2}\right)}{g^{\prime 2}\left(q^{2}\right)}\right] \\
& +\left(\frac{g^{2}\left(m_{\tilde{f}}^{2}\right)}{8 \pi^{2}} I_{e_{\alpha}^{-}}\left(I_{e_{\alpha}^{-}}+1\right)+\frac{g^{\prime 2}\left(m_{\tilde{f}}^{2}\right)}{8 \pi^{2}} \frac{Y_{e_{\alpha}^{-}}^{2}}{4}\right) 3 \ln \frac{q^{2}}{m_{\tilde{f}}^{2}} \\
& -\frac{g^{2}\left(m_{\tilde{f}}^{2}\right)}{4 \pi^{2}} I_{\tilde{f}_{\beta}}\left(I_{\tilde{f}_{\beta}}+1\right)\left[\frac{1}{c} \ln \frac{q^{2}}{m_{\tilde{f}}^{2}}\left(\ln \frac{g^{2}\left(m_{\tilde{f}}^{2}\right)}{g^{2}\left(q^{2}\right)}-1\right)+\frac{1}{c^{2}} \ln \frac{g^{2}\left(m_{\tilde{f}}^{2}\right)}{g^{2}\left(q^{2}\right)}\right] \\
& -\frac{g^{\prime 2}\left(m_{\tilde{f}}^{2}\right) Y_{\tilde{f}_{\beta}}^{2}}{16 \pi^{2}}\left[\frac{1}{c^{\prime}} \ln \frac{q^{2}}{m_{\tilde{f}}^{2}}\left(\ln \frac{g^{\prime 2}\left(m_{\tilde{f}}^{2}\right)}{g^{\prime 2}\left(q^{2}\right)}-1\right)+\frac{1}{c^{\prime 2}} \ln \frac{g^{\prime 2}\left(m_{\tilde{f}}^{2}\right)}{g^{\prime 2}\left(q^{2}\right)}\right] \\
& +\left(\frac{g^{2}\left(m_{\tilde{f}}^{2}\right)}{8 \pi^{2}} I_{\tilde{f}_{\beta}}\left(I_{\tilde{f}_{\beta}}+1\right)+\frac{g^{\prime 2}\left(m_{\tilde{f}}^{2}\right)}{8 \pi^{2}} \frac{Y_{\tilde{f}_{\beta}}^{2}}{4}\right) 4 \ln \frac{q^{2}}{m_{\tilde{f}}^{2}}+\tilde{\delta}_{e_{-\alpha}^{+} e_{\alpha}^{-} \longrightarrow \overline{\tilde{f}}_{-\beta} \tilde{f}_{\beta}} \ln \frac{q^{2}}{m_{\tilde{f}}^{2}} \\
& -\frac{g^{2}\left(m_{\tilde{f}}^{2}\right)}{8 \pi^{2}} \ln \frac{q^{2}}{m_{\tilde{f}}^{2}}\left[\left(\tan ^{2} \theta_{\mathrm{w}} Y_{e_{\alpha}^{-}} Y_{\tilde{f}_{\beta}}+4 T_{e_{\alpha}^{-}}^{3} T_{\tilde{f}_{\beta}}^{3}\right) \ln \frac{t}{u}\right. \\
& \left.\left.+\frac{\delta_{\alpha, \mathrm{L}} \delta_{\beta, \mathrm{L}}}{\tan ^{2} \theta_{\mathrm{w}} Y_{e_{\alpha}^{-}} Y_{\tilde{f}_{\beta}} / 4+T_{e_{\alpha}^{-}}^{3} T_{\tilde{f}_{\beta}}^{3}}\left(\delta_{d, \tilde{f}} \ln \frac{-t}{q^{2}}-\delta_{u, \tilde{f}} \ln \frac{-u}{q^{2}}\right)\right]\right\}
\end{aligned}
$$

where the last line only contributes for left handed (L) electrons and the $d, u$ symbols denote the corresponding isospin quantum number of $\tilde{f}$. In addition we denote $c=\frac{g^{2}\left(m_{\tilde{f}}^{2}\right) \tilde{\beta}_{0}}{4 \pi^{2}}$, $c^{\prime}=\frac{g^{\prime 2}\left(m_{\tilde{f}}^{2} \tilde{\beta}_{0}^{\prime}\right.}{4 \pi^{2}}$, where $g, g^{\prime}$ are the $S U(2)_{l} \times U(1)$ gauge couplings. Here we assume that the asymptotic MSSM $\beta$ functions can be used with

$$
\begin{aligned}
\tilde{\beta}_{0} & =\frac{3}{4} C_{A}-\frac{n_{g}}{2}-\frac{n_{h}}{8}, \tilde{\beta}_{0}^{\prime}=-\frac{5}{6} n_{g}-\frac{n_{h}}{8} \\
g^{2}\left(q^{2}\right) & =\frac{g^{2}\left(m_{\tilde{f}}^{2}\right)}{1+\tilde{\beta}_{0} \frac{g^{2}\left(m_{\tilde{f}}^{2}\right)}{4 \pi^{2}} \ln \frac{q^{2}}{m_{\tilde{f}}^{2}}}, g^{\prime 2}\left(q^{2}\right)=\frac{g^{\prime 2}\left(m_{\tilde{f}}^{2}\right)}{1+\tilde{\beta}_{0}^{\prime} \frac{g^{\prime 2}\left(m_{\tilde{f}}^{2}\right)}{4 \pi^{2}} \ln \frac{q^{2}}{m_{\tilde{f}}^{2}}}
\end{aligned}
$$

where $C_{A}=2, n_{g}=3$ and $n_{h}=2$. In practice, one has to use the relevant numbers of active particles in the loops. These terms correspond to the RG-SL corrections just as in the case of the SM as discussed in Refs. [6] but now with the MSSM particle spectrum contributing. They originate only from $R G$ terms within loops which without the RG contribution would give a DL correction. It must be mentioned that the one-loop RG corrections do not exponentiate and are omitted in the above expression! They are, however, completely determined by the renormalization group in softly broken supersymmetric theories such as the MSSM and sub-subleading at the higher than one loop order.

The generic term denoted by $\tilde{\delta}_{e_{-\alpha}^{+} e_{\alpha}^{-} \longrightarrow \tilde{f}_{-\beta} \tilde{f}_{\beta}}$ in Eq. (3.6) is a short hand notation for the overall (initial and final) one loop SUSY corrections discussed in the previous section. The 
result in Eq. (3.6) is given for the chiral superpartners directly. In principle, mixing effects need to be taken into account for the mass eigenstates of the third family as discussed in Sect.IV.

Eq. (3.6) contains all SL terms to all orders under the assumptions stated above. In particular it provides an independent check on the diagrammatic one-loop results derived in Sect.2 for all universal corrections from gauge bosons as well as the angular dependent terms.

As mentioned above, to compare the asymptotic expansion (3.6) (valid in an energy regime where the details of electroweak symmetry breaking can be neglected) with a physical one-loop calculation like the one that we performed in Sect.II, a number of "minor" adjustments must be performed. In practice, one should use, rather than a common mass $m_{\tilde{f}}$, the gauge boson masses $M_{W}, M_{Z}$ or the SUSY mass $M_{S U S Y}$ in the corresponding logarithms. After these replacements, the one-loop version of Eq. (3.6) should reproduce the corresponding results of Sect.II.

We have verified that the asymptotic expansion Eq. (3.6) and the corresponding physical one-loop expressions of Sect.II do actually coincide. This can be verified in a reasonably simple way, which we do not show in detail here to avoid writing extra long equations. The result can be considered, in our opinion, a satisfactory check of both the various theoretical arguments presented in this Section and of the detailed calculations of Sect.II.

In the case of charged Higgs production we have analogously:

$$
\begin{aligned}
d \sigma_{e_{-\alpha}^{+} e_{\alpha}^{-} \longrightarrow}^{\mathrm{SL}} & H^{+} H^{-}=d \sigma_{e_{-\alpha}^{+} e_{\alpha}^{-} \longrightarrow H^{+} H^{-}}^{\text {Born }} \\
& \exp \left\{-\frac{g^{2}\left(m_{H}^{2}\right)}{4 \pi^{2}} I_{e_{\alpha}^{-}}\left(I_{e_{\alpha}^{-}}+1\right)\left[\frac{1}{c} \ln \frac{q^{2}}{m_{H}^{2}}\left(\ln \frac{g^{2}\left(m_{H}^{2}\right)}{g^{2}\left(q^{2}\right)}-1\right)+\frac{1}{c^{2}} \ln \frac{g^{2}\left(m_{H}^{2}\right)}{g^{2}\left(q^{2}\right)}\right]\right. \\
& -\frac{g^{\prime 2}\left(m_{H}^{2}\right) Y_{e_{\alpha}^{-}}^{2}}{16 \pi^{2}}\left[\frac{1}{c^{\prime}} \ln \frac{q^{2}}{m_{H}^{2}}\left(\ln \frac{g^{\prime 2}\left(m_{H}^{2}\right)}{g^{\prime 2}\left(q^{2}\right)}-1\right)+\frac{1}{c^{\prime 2}} \ln \frac{g^{\prime 2}\left(m_{H}^{2}\right)}{g^{\prime 2}\left(q^{2}\right)}\right] \\
& +\left(\frac{g^{2}\left(m_{H}^{2}\right)}{8 \pi^{2}} I_{e_{\alpha}^{-}}\left(I_{e_{\alpha}^{-}}+1\right)+\frac{g^{\prime 2}\left(m_{H}^{2}\right)}{8 \pi^{2}} \frac{Y_{e_{\alpha}^{-}}^{2}}{4}\right) 3 \ln \frac{q^{2}}{m_{H}^{2}} \\
& -\frac{g^{2}\left(m_{H}^{2}\right)}{4 \pi^{2}} I_{H}\left(I_{H}+1\right)\left[\frac{1}{c} \ln \frac{q^{2}}{m_{H}^{2}}\left(\ln \frac{g^{2}\left(m_{H}^{2}\right)}{g^{2}\left(q^{2}\right)}-1\right)+\frac{1}{c^{2}} \ln \frac{g^{2}\left(m_{H}^{2}\right)}{g^{2}\left(q^{2}\right)}\right] \\
& -\frac{g^{\prime 2}\left(m_{H}^{2}\right) Y_{H}^{2}}{16 \pi^{2}}\left[\frac{1}{c^{\prime}} \ln \frac{q^{2}}{m_{H}^{2}}\left(\ln \frac{g^{\prime 2}\left(m_{H}^{2}\right)}{g^{\prime 2}\left(q^{2}\right)}-1\right)+\frac{1}{c^{\prime 2}} \ln \frac{g^{\prime 2}\left(m_{H}^{2}\right)}{g^{\prime 2}\left(q^{2}\right)}\right] \\
& \left.+\frac{g^{2}\left(m_{H}^{2}\right)}{8 \pi^{2}} I_{H}\left(I_{H}+1\right)+\frac{g^{\prime 2}\left(m_{H}^{2}\right)}{8 \pi^{2}} \frac{Y_{H}^{2}}{4}\right) 4 \ln \frac{q^{2}}{m_{H}^{2}}+\tilde{\delta}_{e_{-\alpha}^{+} e_{\alpha}^{-} \longrightarrow H^{+} H^{-}} \ln \frac{q^{2}}{m_{H}^{2}} \\
& \left.-\frac{g^{2}\left(m_{H}^{2}\right)}{4 \pi^{2}} \ln \frac{q^{2}}{m_{H}^{2}}\left[\delta_{\alpha, \mathrm{L}}\left(\frac{1}{2 c_{\mathrm{w}}^{2}} \ln \frac{t}{u}+2 c_{\mathrm{w}}^{2} \ln \frac{-t}{q^{2}}\right)+\delta_{\alpha, \mathrm{R}} \tan ^{2} \theta_{\mathrm{w}} \ln \frac{t}{u}\right]\right\}
\end{aligned}
$$

where we denote $c=\frac{g^{2}\left(m_{H}^{2}\right) \tilde{\beta}_{0}}{4 \pi^{2}}, c^{\prime}=\frac{g^{\prime 2}\left(m_{H}^{2}\right) \tilde{\beta}_{0}^{\prime}}{4 \pi^{2}}$.

The generic term denoted by $\tilde{\delta}_{e_{-\alpha}^{+} e_{\alpha}^{-} \longrightarrow H^{+} H^{-}}$in Eq. (3.9) is again a short hand notation for the one loop SUSY corrections discussed in the previous section.

We emphasize again the independent nature of deriving the remaining results in Eq. (3.9) as they are directly obtained from $e^{+} e^{-} \rightarrow \phi^{+} \phi^{-}$[2,6] if we neglect the mass-difference. A 
comparison with the one loop calculation confirms again the corresponding contributions of the explicit diagrammatic calculation of Sect.II.

Having checked the equality of the asymptotic resummed subleading expansion and of the physical one loop calculation for all SUSY production processes (modulo $\tilde{e}, \tilde{\nu_{e}}$ to be considered later on), in the next sections we turn to a discussion of the phenomenological importance of the one loop and resummed corrections particularly for a determination of $\tan \beta$.

\section{NUMERICAL RESULTS}

Given the fact that we have now at our disposal both a physical one-loop calculation and a consistent SL order resummation, we are in a position to compute all relevant observables in the two approximations and to identify, whenever this occurs, the importance of the extra resummation in the MSSM case.

To summarize the numerical weight of the various contributions to the one loop cross sections

$$
e^{+} e^{-} \rightarrow \widetilde{f}_{L, R} \tilde{f}_{L, R}^{*}, H^{+} H^{-}
$$

we approximate the previous results by replacing in the logarithms $M_{W}, M_{\gamma}$ and $M_{\gamma Z}$ with $M_{Z}$ and by using everywhere a single SUSY mass scale $M_{S U S Y}$ in place of the various $M_{\chi^{+}}$, $M_{\chi^{0}}$ and $M_{\widetilde{f}}$. Under this approximation, we obtain

$$
\begin{aligned}
100 \frac{\delta \sigma}{\sigma} & =\left(k_{S M}^{R G}+k_{S U S Y}^{R G}\right) \ln \frac{q^{2}}{\mu^{2}}+ \\
& +k_{\text {gauge }}^{\text {in }} L_{3}\left(\frac{q^{2}}{M_{Z}^{2}}\right)+k_{S U S Y}^{\text {in }} \ln \frac{q^{2}}{M_{S}^{2}}+ \\
& +k_{\text {gauge }}^{\text {fin }} L_{4}\left(\frac{q^{2}}{M_{Z}^{2}}\right)+k_{S U S Y}^{\text {fin }} \ln \frac{q^{2}}{M_{S}^{2}}+ \\
& +\left(k_{t}^{Y u k} \frac{m_{t}^{2}}{M_{W}^{2} \sin ^{2} \beta}+k_{b}^{Y u k} \frac{m_{b}^{2}}{M_{W}^{2} \cos ^{2} \beta}\right) \ln \frac{q^{2}}{M_{S}^{2}}+k^{B o x} \ln \frac{q^{2}}{M_{Z}^{2}}
\end{aligned}
$$

where

$$
L_{n}(x)=n \ln x-\ln ^{2} x
$$

and $k_{S M}^{R G}, k_{S U S Y}^{R G}, k_{\text {gauge }}^{\text {in }}, k_{S U S Y}^{i n}, k_{\text {gauge }}^{f i n}, k_{S U S Y}^{f i n}, k_{t, b}^{Y u k}, k^{B o x}$ are numerical coefficients. Their detailed origin is the following

$-k_{S M}^{R G}, k_{S U S Y}^{R G}:$ Renormalization Group terms, Eqs.(2.20,2.21).

- $k_{\text {gauge }}^{\text {in }}, k_{S U S Y}^{\text {in }}$ : initial gauge and SUSY terms according to Eqs.(2.26,2.30).

$-k_{\text {gauge }}^{\text {fin }}$ : final gauge terms, namely the $\theta$ independent logarithms in Eqs.(2.31,2.32) with $M_{W}$ set to $M_{Z}$. 
$-k_{S U S Y}^{f i n}$ : final SUSY terms; these are the $\tan \beta$ independent terms appearing in Eqs.(2.39-2.43).

- $k_{t, b}^{Y u k}$ : final "massive" SUSY terms; these are the $\tan \beta$ dependent terms proportional to $1 / \sin ^{2} \beta$ and $1 / \cos ^{2} \beta$ respectively in Eqs. 2.39 2.43).

$-k^{B o x}$ : all the terms resulting from the angular integration of the $\theta$ dependent terms (note that we are now separating box terms from final gauge ones, differently from what we did in eqs.(2.31)-(2.37) ).

The following remarkable relations hold

$$
\begin{gathered}
k_{\text {gauge }}^{\text {in }}=-k_{S U S Y}^{i n} \stackrel{\text { def }}{=} k^{i n} \\
k_{S U S Y}^{\text {fin }}=-2 k_{\text {gauge }}^{\text {fin }}
\end{gathered}
$$

and allow to write the simpler expression

$$
\begin{aligned}
100 \frac{\delta \sigma}{\sigma} & =\left(k_{S M}^{R G}+k_{S U S Y}^{R G}\right) \ln \frac{q^{2}}{\mu^{2}}+ \\
& +k^{i n}\left[L_{3}\left(\frac{q^{2}}{M_{Z}^{2}}\right)-\ln \frac{q^{2}}{M_{S}^{2}}\right]+k_{g a u g e}^{\text {fin }}\left[L_{4}\left(\frac{q^{2}}{M_{Z}^{2}}\right)-2 \ln \frac{q^{2}}{M_{S}^{2}}\right]+ \\
& +\left(k_{t}^{Y u k} \frac{m_{t}^{2}}{M_{W}^{2} \sin ^{2} \beta}+k_{b}^{Y u k} \frac{m_{b}^{2}}{M_{W}^{2} \cos ^{2} \beta}\right) \ln \frac{q^{2}}{M_{S}^{2}}+k^{B o x} \ln \frac{q^{2}}{M_{Z}^{2}}
\end{aligned}
$$

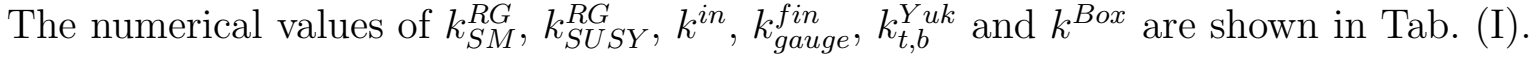

In the case of the longitudinal and forward-backward asymmetries we compute instead the absolute percentual effect $100 \delta A$ and group the various contributions exactly as in the previous equation. The numerical values of the coefficients for $A_{L R}$ are also shown in Tab. (III). In the case of $A_{F B}$, only $k^{B o x}$ is non zero and its values are

$$
A_{F B}: \quad k^{B o x}=\left\{\begin{array}{ccccc}
\tilde{t}_{L} & \widetilde{t}_{R} & \widetilde{b}_{L} & \widetilde{b}_{R} & H^{ \pm} \\
-1.3 & -0.32 & 1.3 & 0.16 & 1.1
\end{array}\right.
$$

We shall now illustrate the main results of our analysis in the following figures. We must anticipate at this point that the qualitative features of the various effects show a strong difference between the two cases of

a) production of sleptons in general (with the exclusion of selectrons and $\tilde{\nu_{e}}$ ) and squarks of the first two families and

b) production of squarks of the third family and of charged Higgs bosons. One can already guess that the difference will be due to the appearance, in the second case, of contributions of SUSY Yukawa type, that will give rise to relevant and possibly interesting effects to be discussed later on.

Fig.6 shows the relative logarithmic effects at one loop (dashed line) and after resummation (full line) for $\tilde{\mu}_{L, R}, \tilde{\tau}_{L, R}, \tilde{\nu}_{\mu, L}, \tilde{\nu}_{\tau, L}$, cross sections at variable energy, while in Fig.7 the same effects are shown for the first two families of squarks (denoted as $\tilde{u}, \tilde{d}$. Figs.8.9. 
correspond to the cases of the forward-backward asymmetries of the same processes. In all Figures, the "logarithmic" effect takes into account both the RG SL and the Sudakov DL,SL terms.

As one sees from Figs.66, the general feature is that the logarithmic effect at one loop in the energy region between $1 \mathrm{TeV}$ and $2 \mathrm{TeV}$ is very close to the resummed effect, up to less than one percent differences that in our working assumption should be experimentally invisible. This feature, that is valid for both cross sections and forward-backward asymmetries, is intuitively related to the fact that the one-loop effects are in these cases relatively "small", although experimentally meaningful, being typically of several percent size. The same conclusions apply to the cases of the longitudinal polarization asymmetries of this first class of processes. In order to avoid a too large number of Figures, we have given the corresponding effects in Tables 2,3. The situation changes when one moves to larger energies, $2 \mathrm{TeV} \lesssim \sqrt{q^{2}} \lesssim 4 \mathrm{TeV}$ (the latter value is chosen as an optimistic "averaged" aim for CLIC experiments). Here, for left-handed sleptons and squarks, the difference between one loop and resummation becomes appreciable (beyond the one percent level) and a complete resummation appears necessary.

The conclusion of this first investigation is that, in the energy range of the TeV size, assuming a typical SUSY mass of few hundred GeV, the perturbative treatment of SUSY scalar production for the considered set of processes appears to us to be totally satisfactory at the one-loop level, not requiring extra resummations if the aimed experimental accuracy remains at the one percent (and not at the one permille) level (we stress that, if the SUSY mass turned out to be larger, these conclusions can be simply rescaled at correspondingly larger c.m. energies). For larger energies, as resummation seems to be requested for lefthanded scalars.

As a side remark that might be added, we have also depicted in Figs.6-9 the separate resummed effects that would have been obtained by ignoring the SL $\theta$-dependent contributions of box origin. As one sees, the consequence of this omission would have been catastrophic in the left sfermion cases, in particular, the angular terms are the only ones that contribute the forward-backward asymmetries. This confirms a previous observation [8] that stressed the relevance of these "box-type" contributions in the Sudakov regime.

When we move to the production of either third family of squarks or charged Higgs bosons (we remind that neutral Higgs bosons production will be treated in detail in a forthcoming paper), a different picture arises. A priori, we know that SUSY Yukawa contributions will be effective in these cases, and they will depend on the $\tan \beta$ parameter. This statement is only true for cross sections, and does not hold for forward-backward asymmetries, since the latter ones are not affected by universal, $\theta$-independent, contributions. To evidentiate whether this expectation is correct, we have depicted in Figs.10, 12. a the effects on the cross sections for squarks and Higgs bosons production at two representative and sensibly different values $\tan \beta=10$ and $\tan \beta=40$. Again, we have drawn systematically the one loop (dashed) and resummed (full) effects. In Figs.11,12 $\mathrm{b}$ we have drawn the forward-backward asymmetries of Higgs bosons and squarks production that, as we said, do not depend on $\tan \beta$. The first characteristic feature of Figs.10,12a is that now a more drastic difference exists between the energy region $\sqrt{q^{2}} \simeq 1 \mathrm{TeV}$ (possibly within the final aimed reach of LC) and that of $\sqrt{q^{2}} \simeq 3-4 \mathrm{TeV}$ (possibly within the CLIC range). In the first case, the same previous conclusions about the reliability of a one-loop expansions are still, in our working 
assumptions, essentially valid (in fact, the relative difference between the one-loop and the resummed effects is always below the assumed visible one percent level. This reliability is totally lost when one reaches the $\simeq 3 \mathrm{TeV}$ regime. In this case, the relative difference between the two effects is well beyond the one percent level, particularly for large $\tan \beta$. In the extreme case of Higgs boson production at $\tan \beta=40$, the relative difference between the two effects is of approximately five percent at $3 \mathrm{TeV}$. Similar features are valid for squark production as well. Thus, for third family squarks and charged Higgs boson production in the CLIC energy regime, with a SUSY mass of few hundred TeV, stopping the theoretical calculation at one loop level would be in our opinion a theoretical catastrophe. It should also be stressed that the resummed effect remains large and sometimes spectacular. In particular, in the extreme case of charged Higgs bosons production at $\tan \beta=40$, it reaches the 35 percent value at $\sqrt{q^{2}}=3 \mathrm{TeV}$. For squarks, the effect is reduced but is still generally large (from $\simeq 5$ to $\simeq 15$ depending on the cases). We insist again on the fact that, as a consequence of the color factor in the quark loop in Fig.2c, the process of charged Higgs production exhibits the most sizeable SUSY Yukawa Sudakov effect, as we anticipated in Sect.II.

In the case of forward-backward asymmetries, depicted in Figs.11, 12]b , the situation is very similar to that of the sleptons and first families of squarks, as one can see. The only difference appears in the case of Higgs boson production. Here the difference between oneloop and resummed effects becomes again visible in the CLIC (but not in the LC) regime. This fact is, in our opinion, accidental since we could not find deeper physical motivations for it.

The second relevant feature of Figs.10,12a is the fact that a strong dependence on $\tan \beta$ appears in the logarithmic effect. This can be seen by comparison of the two curves that correspond to $\tan \beta=10$ and $\tan \beta=40$. As one notices, the relative difference between the two effects is always "large" (depending on the case again with an enhancement in the Higgs boson case), with the only exception of right stop production (this can be qualitatively understood looking at Eq.(2.40)). This remarkable feature remains essentially true in the overall 1-4 TeV energy range, although it increases with energy. The previous observation suggests that, from a special analysis of cross sections, one might be able to derive interesting informations on $\tan \beta$. In fact, this possibility was already considered in a previous paper devoted to top quark production [20], where it was proposed to exploit measurements of the slope of the cross section to fix the $\tan \beta$ value. In the next Sect.V we shall generalize the previous proposal to the cases of stop, sbottom, charged Higgs boson production, and discuss in some detail the possible consequences for a relative precise determination of $\tan \beta$.

A final comment has to be added concerning the mixing effects which affect the third family of squarks. The mass eigenstates will no more be $\tilde{f}_{L, R}$ but the combinations $\tilde{f}_{1}=$ $\cos \theta_{f} \tilde{f}_{L}+\sin \theta_{f} \tilde{f}_{L}$ and $\tilde{f}_{2}=-\sin \theta_{f} \tilde{f}_{L}+\cos \theta_{f} \tilde{f}_{L}$. Experimental results will be obtained for production of $\tilde{f}_{1} \tilde{\bar{f}}_{1}, \tilde{f}_{2} \tilde{\bar{f}}_{2}, \tilde{f}_{1} \tilde{\bar{f}}_{2}, \tilde{f}_{2} \tilde{\bar{f}}_{1}$. However in the asymptotic regime regime, the amplitudes for mass eigenstates can be expressed in terms of amplitudes for chiral $L, R$ states in a simple way:

$$
\begin{aligned}
& A_{11}=A_{L L} \cos ^{2} \theta_{f}+A_{R R} \sin ^{2} \theta_{f} \\
& A_{22}=A_{L L} \sin ^{2} \theta_{f}+A_{R R} \cos ^{2} \theta_{f}
\end{aligned}
$$




$$
A_{12}=A_{21}=\left(A_{R R}-A_{L L}\right) \sin \theta_{f} \cos \theta_{f}
$$

so that it should be straightforward to express the experimental results in terms of the observables concerning the chiral states that we considered in this paper. One has just to invert the above equations, and one obtains:

$$
\begin{gathered}
\tan 2 \theta_{f}=\frac{2 A_{12}}{A_{22}-A_{11}} \\
A_{L L}=\frac{A_{11} \cos ^{2} \theta_{f}-A_{22} \sin ^{2} \theta_{f}}{\cos ^{2} \theta_{f}-\sin ^{2} \theta_{f}} \\
A_{R R}=\frac{A_{22} \cos ^{2} \theta_{f}-A_{11} \sin ^{2} \theta_{f}}{\cos ^{2} \theta_{f}-\sin ^{2} \theta_{f}}
\end{gathered}
$$

for each flavor $\tilde{t}$ or $\tilde{b}$, separately. The experimental results can then be given for chiral states and compared with the theoretical predictions made throughout this paper.

\section{DETERMINATION OF $\tan \beta$}

In the framework of the MSSM, we showed in a previous paper [13] that the cross section for the process $e^{+} e^{-} \rightarrow q \bar{q}$, with $q$ being a third generation quark (top and bottom), contains angular independent Sudakov logarithms of Yukawa origin. These are terms depending on $\tan \beta$ and on the SUSY mass $M_{S U S Y}$, which are the only SUSY parameters surviving in the high energy limit of this process.

To understand the reason for this peculiar feature we recall that the free parameters of the MSSM can be broadly divided into three classes: (i) the ones belonging to the SM sector, (ii) $\tan \beta$ that is related to the two doublet structure of the Higgs sector and (iii) the (many) SUSY soft breaking mass terms.

As we discussed in [13], an analysis of the slope of the effects in the observables as the energy is increased allows to extract the value of $\tan \beta$ without any specific knowledge of the other parameters. This very welcome feature is present also in the processes considered in this paper; to be more precise, it remains rigorously true when working in the one loop approximation, and it is valid to subleading order accuracy if one uses the complete resummed expressions. The remaining part of this Section will be devoted to a numerical analysis with the specific aim of determining $\tan \beta$.

Let us denote by $\sigma_{n}, n=1-5$ the various cross sections for production of $\widetilde{t}_{L, R}, \widetilde{b}_{L, R}$ or charged Higgs bosons $H^{ \pm}$. We define the relative SUSY effect on the cross section $\sigma_{n}$ as the ratio

$$
\epsilon_{n}\left(q^{2}\right)=\frac{\mathcal{O}_{n}\left(q^{2}\right)-\mathcal{O}_{n}^{S M}\left(q^{2}\right)}{\mathcal{O}_{n}^{S M}\left(q^{2}\right)} .
$$

This definition is useful as far as we can regard the SM contributions as perfectly known terms. As we have already discussed, at energies around $1 \mathrm{TeV}$, this statement is certainly 
true because a one loop calculation is perfectly reliable and reproduces the full effect, with resummation giving a negligible additional shift in the observables.

At one loop, in the asymptotic regime, we can parametrize $\epsilon_{n}$ as

$$
\epsilon_{n}\left(q^{2}\right)=F_{n}(\tan \beta) \ln \frac{q^{2}}{M_{S}^{2}}+G_{n}+\cdots .
$$

Here, $F_{n}$ is a function of $\tan \beta$ only. The explicit form of its $\tan \beta$ dependent terms can be obtained from the Yukawa terms and we write them here for clarity and convenience of the reader

$$
\begin{gathered}
F_{\widetilde{t}_{L}}=-\frac{\alpha}{\pi} \frac{1}{4 M_{W}^{2} s_{W}^{2}}\left(\frac{m_{t}^{2}}{\tan ^{2} \beta}+m_{b}^{2} \tan ^{2} \beta\right) ; \\
F_{\widetilde{t}_{R}}=-\frac{\alpha}{\pi} \frac{1}{2 M_{W}^{2} s_{W}^{2}} \frac{m_{t}^{2}}{\tan ^{2} \beta} ; \\
F_{\widetilde{b}_{R}}=-\frac{\alpha}{\pi} \frac{1}{2 M_{W}^{2} s_{W}^{2}} m_{b}^{2} \tan ^{2} \beta ; \\
F_{H^{ \pm}}=-\frac{\alpha}{\pi} \frac{3}{4 M_{W}^{2} s_{W}^{2}}\left(\frac{m_{t}^{2}}{\tan ^{2} \beta}+m_{b}^{2} \tan ^{2} \beta\right) .
\end{gathered}
$$

The constant $G_{n}$ in Eq. (5.2) is a sub-subleading correction. It does not increase with $q^{2}$, but depend on all mass ratios of virtual particles. The omitted terms in Eq. (5.2) vanish in the high energy limit.

To eliminate $G_{n}$ we proceed as in 13]. We assume that a set of $N$ independent measurements is available at c.m. energies $\sqrt{q_{1}^{2}}, \sqrt{q_{2}^{2}}, \ldots, \sqrt{q_{N}^{2}}$ and take the difference of each measurement with respect to the one at lowest energy. The resulting quantities

$$
\delta_{n, i}=\epsilon_{n}\left(q_{i}^{2}\right)-\epsilon_{n}\left(q_{1}^{2}\right)
$$

do not contain $G_{n}$ and take the simple form

$$
\delta_{n, i}=F_{n}\left(\tan \beta^{*}\right) \ln \frac{q_{i}^{2}}{q_{1}^{2}}
$$

where $\tan \beta^{*}$ is the true unknown value that describes the experimental measurements.

We now describe how precisely $\tan \beta$ can be extracted. We denote by $\sigma_{n}\left(q^{2}\right)$ the experimental error on $\epsilon_{n}\left(q^{2}\right)$. For each set of explicit measurements $\left\{\delta_{n}\left(q_{i}^{2}\right)\right\}$, the best estimate for $\tan \beta$ is the value that minimizes the $\chi^{2}$ sum

$$
\chi^{2}(\tan \beta)=\sum_{i=1}^{N} \sum_{n=1}^{N_{\mathcal{O}}} \frac{\left[F_{n}(\tan \beta) \ln \frac{q_{i+1}^{2}}{q_{1}^{2}}-\delta_{n, i}\right]^{2}}{4 \sigma_{n, i}^{2}}
$$


where $\delta_{n, i} \equiv \delta_{n}\left(q_{i}^{2}\right)$ and $\sigma_{n, i} \equiv \sigma_{n}\left(q_{i}^{2}\right)$.

As usual, the experimentally measured quantity $\delta_{n, i}$ is assumed to be a normal Gaussian random variable distributed around the value

$$
F_{n}\left(\tan \beta^{*}\right) \ln \frac{q_{i+1}^{2}}{q_{1}^{2}},
$$

with standard deviation $2 \sigma_{n, i}$. After linearization around $\tan \beta=\tan \beta^{*}$, minimization of $\chi^{2}$ provides the best estimate of $\tan \beta$. This is an unbiased Gaussian estimation with mean $\tan \beta^{*}$ and standard deviation fixed by the condition $\Delta \chi^{2}=1$, i.e.

$$
\delta \tan \beta=2\left(\sum_{n, i}\left(\frac{F_{n}^{\prime}\left(\tan \beta^{*}\right) \ln \frac{q_{i+1}^{2}}{q_{1}^{2}}}{\sigma_{n, i}}\right)^{2}\right)^{-1 / 2} .
$$

Under the simplifying assumption $\sigma_{n, i} \equiv \sigma$, this formula reduces to

$$
\delta \tan \beta=2 \sigma\left(\sum_{n} F_{n}^{\prime}\left(\tan \beta^{*}\right)^{2}\right)^{-1 / 2}\left(\sum_{i} \ln ^{2} \frac{q_{i+1}^{2}}{q_{1}^{2}}\right)^{-1 / 2} .
$$

The function

$$
\tau(\tan \beta)=\left(\sum_{n} F_{n}^{\prime}(\tan \beta)^{2}\right)^{-1 / 2},
$$

measures the dependence of the slope of SUSY effects on $\tan \beta$. It is shown in Fig.13 for four possible choices: (i) all the five cross sections, (ii) without production of $\widetilde{t}_{R}$, (iii) without production of $\widetilde{b}_{R}$, (iv) without production of charged Higgses $H^{ \pm}$.

In the best case (i), it is strongly peaked around $\tan \beta=6$ and the combination of the various observables, especially the cross sections for production of right sfermions (the ones with larger $\cot ^{2} \beta$ coefficient) is crucial to keep the function $\tau(\tan \beta)$ as small as possible.

To understand the consequences of the shape of $\tau$, we plot in Fig.14 the relative error $\delta \tan \beta / \tan \beta$ computed under the optimistic assumption of a relative accuracy equal to $1 \%$ for all the five observables. The three curves correspond to the assumption that independent measurements for each observable are available at $N=10$ equally spaced c.m. energies around $1 \mathrm{TeV}$, between $0.8 \mathrm{TeV}$ and $1.5 \mathrm{TeV}$. We remark that different curves associated to pairs $(N, \sigma)$ depend only on the combination $\sigma / \sqrt{N}$. In the Figure, we also show horizontal dashed lines corresponding to relative errors equal to 1 and 0.5. As one can see in the Figure, values in the range

$$
\tan \beta<3, \quad \tan \beta>16
$$

can be detected with $N=10 \mathrm{c}$.m. energy values with a relative error smaller than $50 \%$, that can be considered qualitatively as a "decent" accuracy. If a higher experimental precision (e.g. a few permille in the cross sections) were achievable, the same result could be obtained with a smaller number $(N \simeq 3)$ of independent energy measurements. The first region $(\tan \beta<3)$ appears to be unfavoured by the present LEP combined data analysis, but the second one $(\tan \beta>14)$ is instead very interesting for the LHC physics programme. 
The extension of the previous analysis to the case of a Linear Collider working at an energy around $3 \mathrm{TeV}$ (CLIC), is not straightforward. In fact, the one loop expressions are no more reliable and a resummation of higher order terms must be performed. Up to date, the best theoretical accuracy that can be reached in the MSSM is the subleading one discussed in this paper. The Yukawa terms are then given at all orders by combining the one loop Yukawa contributions with the resummed double logarithms of gauge origin. To this level of accuracy, the relative SUSY Yukawa effect is thus unchanged and the analysis can be repeated with the same formulae. However, one must keep in mind that the subleading approximation can be enough to determine the gross size of the virtual effects, but its validity must be checked in the analysis of finer details like the dependence on $\tan \beta$ of the omitted constant terms. Notwithstanding these unavoidable remarks, we can analyze what happens at $3 \mathrm{TeV}$ in the subleading approximation.

As we said above, the same expressions as in the $1 \mathrm{TeV}$ analysis can be applied. The need to eliminate any sub-subleading constant from the SUSY effects leads again to Eq. (5.11). The effects thus depend on the logarithms of the ratios between the various measurement energies and the lowest energy $q_{1}$. Therefore the same results on the error $\delta \tan \beta / \tan \beta$ that we derived around $1 \mathrm{TeV}$ can be extended to the rescaled (wider) energy range

$$
[0.8,1.5] \mathrm{TeV} \rightarrow[2.7,4.5] \mathrm{TeV}
$$

with no additional remarks or changes.

It might be interesting to discuss what would be the change in such an analysis if data could be accumulated starting at $800 \mathrm{GeV}$ and increasing the energy up to an upper value around $3 \mathrm{TeV}$. In this case, shown in Fig.15, the results are largely improved with respect to those that could be with measurements at 1 or $3 \mathrm{TeV}$ only. To be more precise, with 10 measurements extending from $800 \mathrm{GeV}$ to $3.3 \mathrm{TeV}$, the region $\tan \beta>11$ can be accessible with $50 \%$ accuracy. This bound reduces to $\tan \beta>14$ if the required accuracy is $25 \%$. We stress that this is a quite interesting region 3 as discussed in [14], where it is shown that a measurement of $\tan \beta$ is practically impossible from chargino or neutralino production when $\tan \beta>10$ since the effects depend on $\cos 2 \beta$ that becomes flat for $\beta \rightarrow \pi / 2$. It could be achieved in the associated productions $e^{+} e^{-} \rightarrow h \tilde{\tau} \tilde{\tau}$ or $e^{+} e^{-} \rightarrow A \bar{b} b$ (with $h$ and $A$ being the $\mathrm{CP}$ even and odd Higgs bosons), but only for very large $\tan \beta$ values $(\sim 50)$.

\section{CONCLUSIONS}

In this final Section we shall draw a number of relevant conclusions. Before doing that, we feel though, that some preliminary considerations are opportune.

In this paper we have derived the complete Sudakov one-loop expansion and the related all orders subleading resummation for SUSY scalar production. Since both expressions have been computed for the first time, there are no different papers in the literature with which to compare our results. It is, however, a highly non-trivial check that all SM DL and SL

\footnotetext{
${ }^{3}$ For completeness, we remark that here too, as in the previous analysis, there is a region at low $\tan \beta$ that can be analyzed.
} 
terms (in particular the angular dependent contributions) are in agreement with the results obtained in the symmetric basis in Sect.III. For the SL terms of genuine supersymmetric origin obtained from the physical one-loop calculation that we have performed, and that has led us to a large number of equations and formulae, no such internal cross check is available.

In spite of the lack of possible comparisons, however, there are a few other internal consistency checks of our results that serve to support their validity. Here we list a limited number of them in the following order:

a) The box diagrams with $2 W$ exchange and the vertex diagrams with initial electron and final scalar $2 W$ triangle get combined separately in a tricky way. One half of the box must be summed with the initial $2 W$ vertex, the other one with the final $2 W$ vertex. After these summations, one gets the correct universal factors $L_{3}=\left(3 \ln -\ln ^{2}\right)$ for the initial state and $L_{4}=\left(4 \ln -\ln ^{2}\right)$ for the final state, although none of the separate ( $W W$ box, $W W$ initial vertex, $W W$ final vertex) contributions produces alone the $L_{3}, L_{4}$ terms.

b) The overall MSSM "gauge" effect for the final scalar pair, obtained by summing the diagrams with SM gauge bosons (i.e. photon, $Z, W$ ) to those with SUSY gauginos, is proportional to a new combination $L_{2}=\left(2 \ln -\ln ^{2}\right)$. This is the same MSSM "gauge" combination, with the same coefficient that affects the respective fermionic superpartners either in the initial or in the final state of $e^{+} e^{-}$annihilation. One can easily check this statement for final sleptons looking at eqs.(2.26),(2.30), (2.33-2.43) of this paper. For final quarks, one should compare the expressions of this paper with the analogous ones given for quark production in the MSSM in a previous paper [12 reducing these expressions to the opportune form (which shall not be explicitly shown here). This equality is expected, a priori, since the contributions are computed for vanishing particles-sparticles masses, where supersymmetry is supposed to be exact. As a consequence, the pure overall gauge effects should be the same within the same supermultiplet.

c) In the MSSM, the final Yukawa effects for stop and sbottom production at lepton colliders are the same as those for top and bottom production. This can be also verified by comparing the expressions of this paper with the corresponding ones of previous references 12,13. This equality is highly non trivial in a technical sense, since in the two cases quite different diagrams contribute (in particular, virtual Higgs bosons vertices do not contribute for squark production, while the corresponding SM scalars do contribute for top, bottom final pairs). We interpret it as a consequence of the MSSM origin of Yukawa couplings, carried by the Higgsino, which is the SUSY superpartner of the Higgs boson and lies therefore in the same supermultiplet.

Having shown the three non trivial consistency checks of our one-loop calculations, we now proceed assuming that our results are correct and draw some final conclusions. Briefly, first of all it seems to us that the process of SUSY scalar production in its Sudakov regime, assuming that the latter coincides with the TeV energy range for reasonably light SUSY masses, can be divided into two distinct categories. The first one includes slepton and first two family squark production. The second one includes the considered third family squark and charged Higgs boson production. For all cases, there is an energy dependence of the one-loop approximation, which begins to be unreliable when one crosses the $\simeq 1,2$ 
$\mathrm{TeV}$ region (where it should still be satisfactory). At CLIC energies, the need of a proper resummation becomes imperative and all our one-loop results must be replaced by our corresponding resummed expressions. The important point is that in this procedure, the (generally) large unreliable one-loop effects are replaced by reliable resummed effects, that are sensibly reduced but still remain large and visible given the expected level of experimental accuracy. Independently of this fact, a strong $\tan \beta$ dependence in the third family and charged Higgs boson production case (particularly effective in the charged Higgs boson case) should allow to achieve, via suitable measurements of the slope of the cross sections in the total energy range $1-4 \mathrm{TeV}$ a satisfactory determination of the SUSY parameter $\tan \beta$. This should be combined with other analogous independent measurements to be performed in other processes (like e.g. neutral Higgs boson production) to obtain a more precise determination (an analysis of this possibility is already in progress [21]).

Finally, we believe to have shown in this example that there exists a realistic energy range, to be hopefully covered in a not too far future, where virtual corrections of the MSSM can become large. Given the previous experience at lower energies in the hundred GeV where SUSY virtual effects were systematically small, we believe that this fact would provide a clean and fundamental test of the validity of the MSSM at future lepton colliders, in case supersymmetric particles were detected somewhere in a (hopefully) near future. 


\section{TABLES}

TABLE I. Numerical values of the $k$ coefficients. They are defined in Eq. (4.6) and represent the relative weights of the various logarithmic one loop effects in the cross sections. The second part of the table shows their numerical values in the case of the $A_{F B}$ asymmetry. A dash denotes a contribution that is rigorously null and not simply numerically negligible.

\begin{tabular}{|c|c|c|c|c|c|c|c|}
\hline $100 \Delta \sigma / \sigma$ & $k_{S M}^{R G}$ & $k_{S U S Y}^{R G}$ & $k^{i n}$ & $k_{\text {gauge }}^{\text {fin }}$ & $k_{t}^{Y u k}$ & $k_{b}^{Y u k}$ & $k^{B o x}$ \\
\hline$\tilde{t}_{L}$ & -1.3 & 1.9 & 0.41 & 0.38 & -0.25 & -0.25 & 1.5 \\
\hline$\widetilde{t}_{R}$ & 1.0 & 0.63 & 0.20 & 0.067 & -0.51 & - & - \\
\hline$\widetilde{b}_{L}$ & -1.7 & 2.2 & 0.40 & 0.38 & -0.25 & -0.25 & 1.8 \\
\hline$\widetilde{b}_{R}$ & 1.0 & 0.63 & 0.20 & 0.017 & - & -0.51 & - \\
\hline$H^{ \pm}$ & -0.63 & 1.6 & 0.37 & 0.41 & -0.76 & -0.76 & 1.1 \\
\hline$\Delta A_{L R}$ & $k_{S M}^{R G}$ & $k_{S U S Y}^{R G}$ & $k^{i n}$ & $k_{\text {gauge }}^{\text {fin }}$ & $k_{t}^{Y u k}$ & $k_{b}^{Y u k}$ & $k^{B o x}$ \\
\hline$\widetilde{t}_{L}$ & -0.15 & 0.083 & 0.016 & - & - & - & 0.095 \\
\hline$\widetilde{t}_{R}$ & - & - & 0.084 & - & - & - & - \\
\hline$\widetilde{b}_{L}$ & -0.26 & 0.15 & 0.024 & - & - & - & 0.17 \\
\hline$\widetilde{b}_{R}$ & - & - & 0.084 & - & - & - & - \\
\hline$H^{ \pm}$ & -0.59 & 0.33 & 0.076 & - & - & - & 0.37 \\
\hline
\end{tabular}


TABLE II. Absolute shift in the asymmetry $A_{L R}$. We consider here two energies 1 and $3 \mathrm{TeV}$. For each energy and final sparticle we show values. "1L" is the one loop contribution, "Res" is the contribution resummed at subleading accuracy. The label "no $\theta$ " means that the genuine non universal angular contributions from boxes have been suppressed. About the sparticle labeling, we remind that $\widetilde{l}$ stands for a generic slepton $\widetilde{\mu}$ or $\widetilde{\tau}$ and that $\widetilde{u}$ and $\widetilde{d}$ stand for squarks with $T^{3}=1 / 2$ and $-1 / 2$ in the first two generation.

\begin{tabular}{l|rrrrrrrr}
\hline \hline $100 \Delta A_{L R}$ & $\begin{array}{r}1 \mathrm{TeV} \\
1 \mathrm{~L}\end{array}$ & $\begin{array}{r}1 \mathrm{TeV} \\
\text { Res }\end{array}$ & $\begin{array}{r}1 \mathrm{TeV} \\
1 \mathrm{~L}, \text { no } \theta\end{array}$ & $\begin{array}{r}1 \mathrm{TeV} \\
\text { Res, no } \theta\end{array}$ & $\begin{array}{r}3 \mathrm{TeV} \\
1 \mathrm{~L}\end{array}$ & $\begin{array}{r}3 \mathrm{TeV} \\
\text { Res }\end{array}$ & $\begin{array}{r}3 \mathrm{TeV} \\
1 \mathrm{~L}, \text { no } \theta\end{array}$ & $\begin{array}{r}3 \mathrm{TeV} \\
\text { Res, no } \theta\end{array}$ \\
\hline & & & & & & & & \\
$\widetilde{l}_{L}$ & -0.27 & -0.17 & -2.4 & -2.3 & -1.9 & -1.6 & -5.7 & -4.8 \\
$\widetilde{l}_{R}$ & -1 & -0.99 & -1 & -0.97 & -3 & -2.6 & -3 & -2.5 \\
$\widetilde{\nu}$ & 0.53 & 0.89 & -6 & -5.9 & -1.5 & -1.3 & -13 & -11 \\
$\widetilde{u}_{L}$ & -0.031 & -0.0069 & -0.57 & -0.56 & -0.36 & -0.31 & -1.4 & -1.2 \\
$\widetilde{u}_{R}$ & -1 & -0.98 & -1 & -0.97 & -2.9 & -2.6 & -2.9 & -2.5 \\
$\widetilde{d}_{L}$ & 0.011 & 0.055 & -0.95 & -0.93 & -0.43 & -0.37 & -2.2 & -1.9 \\
$\widetilde{d}_{R}$ & -1 & -0.98 & -1 & -0.97 & -2.8 & -2.6 & -2.8 & -2.6 \\
$\widetilde{t}_{L}$ & -0.032 & -0.017 & -0.59 & -0.57 & -0.39 & -0.34 & -1.5 & -1.2 \\
$\widetilde{t}_{R}$ & -1.1 & -0.98 & -1.1 & -0.97 & -3.2 & -2.5 & -3.2 & -2.5 \\
$\widetilde{b}_{L}$ & 0.011 & 0.037 & -0.99 & -0.95 & -0.46 & -0.43 & -2.4 & -2 \\
$\widetilde{b}_{R}$ & -1 & -0.97 & -1 & -0.97 & -2.9 & -2.6 & -2.9 & -2.6 \\
$H^{ \pm}$ & -0.3 & -0.3 & -2.6 & -2.4 & -2.4 & -2 & -7.3 & -5.2 \\
\hline \hline
\end{tabular}




\section{REFERENCES}

[1] V. V. Sudakov, Sov. Phys. JETP 3, 65 (1956); Landau-Lifshits: Relativistic Quantum Field theory IV tome (MIR, Moscow) 1972.

[2] W. Beenakker et al, Nucl. Phys. B410, 245 (1993), Phys. Lett. B317, 622 (1993); A. Denner, S. Dittmaier and R. Schuster, Nucl. Phys. B452, 80 (1995); A. Denner, S. Dittmaier and T. Hahn, Phys. Rev. D56, 117 (1997); P. Ciafaloni and D. Comelli, Phys. Lett. B446, 278 (1999); A. Denner, S.Pozzorini; Eur. Phys. J. C18, 461 (2001).

[3] M. Beccaria, P. Ciafaloni, D. Comelli, F. M. Renard and C. Verzegnassi, Phys. Rev. D61, 073005 (2000);

[4] M. Ciafaloni, P. Ciafaloni and D. Comelli, Nucl. Phys. B589, 4810 (2000); V. S. Fadin, L. N. Lipatov, A. D. Martin and M. Melles, Phys. Rev. D61, 094002 (2000); J. H. Kühn, A. A. Penin and V. A. Smirnov, Eur. Phys. J C17, 97 (2000); M. Melles, hepph/0104232; J. H. Kühn, S. Moch, A. A. Penin and V. A. Smirnov, Nucl. Phys. B616, 286 (2001).

[5] M. Melles, PSI-PR-01-11, Aug 2001, hep-ph/0108221.

[6] M. Melles Phys. Rev. D63, 034003 (2001); Phys. Rev. D64, 014011 (2001), Phys. Rev. D64, 054003 (2001).

[7] M. Melles, Phys. Lett. B495, 81 (2000); W. Beenakker, A. Werthenbach, Phys. Lett. B489, 148 (2000); hep-ph/0112030; M. Hori, H. Kawamura and J. Kodaira, Phys. Lett. B491, 275 (2000).

[8] M. Beccaria, F. M. Renard and C. Verzegnassi, Phys. Rev. D64, 073008 (2001).

[9] Opportunities and Requirements for Experimentation at a Very High Energy $e^{+} e^{-}$Collider, SLAC-329(1928); Proc. Workshops on Japan Linear Collider, KEK Reports, 90-2, 91-10 and 92-16; P. M. Zerwas, DESY 93-112, Aug. 1993; Proc. of the Workshop on $e^{+} e^{-}$Collisions at $500 \mathrm{GeV}$ : The Physics Potential, DESY 92-123A,B,(1992), C(1993), D(1994), E(1997) ed. P. Zerwas; E. Accomando et.al. Phys. Rep. C299 (299) 1998.

[10] "The CLIC study of a multi-TeV $e^{+} e^{-}$linear collider", CERN-PS-99-005-LP (1999).

[11] H. Georgi, H. Quinn and S. Weinberg, Phys.Rev.Lett.32,451(1974); S. Dimopoulos, S. Raby and F. Wilczek, Phys. Rev.D24,1681 (1981); L. Ibañez and GG. Ross, Phys.Lett.105B,439(1981).

[12] M. Beccaria, F. M. Renard, C. Verzegnassi, Phys. Rev. D63, 095010, (2001).

[13] M. Beccaria, S. Prelovsek, F. M. Renard, C. Verzegnassi, Phys. Rev. D64, 053016, (2001).

[14] A. Datta, A. Djouadi and J.-L. Kneur, Phys. Lett. B509, 299 (2001).

[15] M. Beccaria, M. Melles, F. M. Renard and C. Verzegnassi, in preparation.

[16] F. M. Renard and C. Verzegnassi, Phys. Rev. D52, 1369 (1995), Phys. Rev. D53, 1290 (1996).

[17] G. Degrassi and A. Sirlin, Nucl. Phys. B383, 73 (1992); Phys. Rev. D46, 3104 (1992).

[18] J. M. Cornwall, Phys. Rev. D26, 1453 (1982); J. M. Cornwall and J. Papavassiliou, ibid 40, 3474 (1989).

[19] J. Rosiek, Phys. Rev. D41, 3464 (1990), hep-ph/9511250 (E).

[20] M. Beccaria, F. M. Renard and C. Verzegnassi, Phys. Rev. D63, 053013 (2001).

[21] M. Beccaria, D. Comelli, M. Melles, F. M. Renard, C. Verzegnassi, paper in preparation. 


\section{FIGURES}

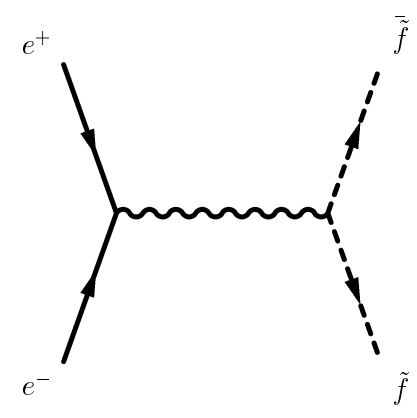

FIG. 1. Born diagram for $e^{+} e^{-} \rightarrow \overline{\tilde{f}} \tilde{f}$

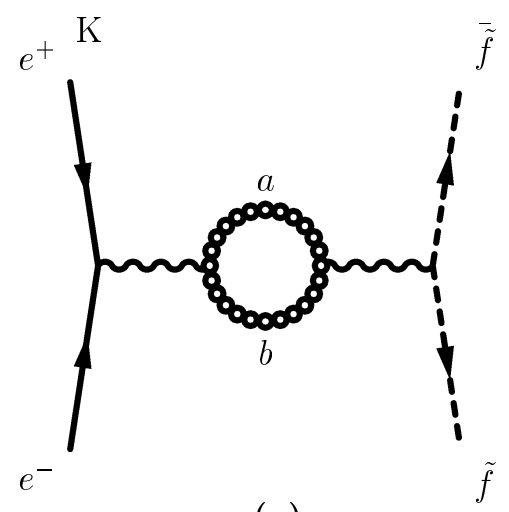

(a)

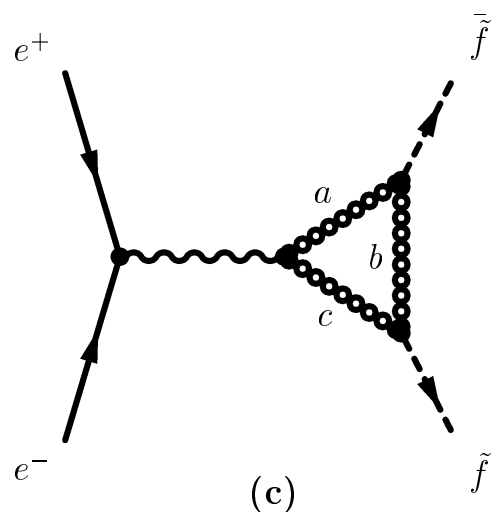

(c)

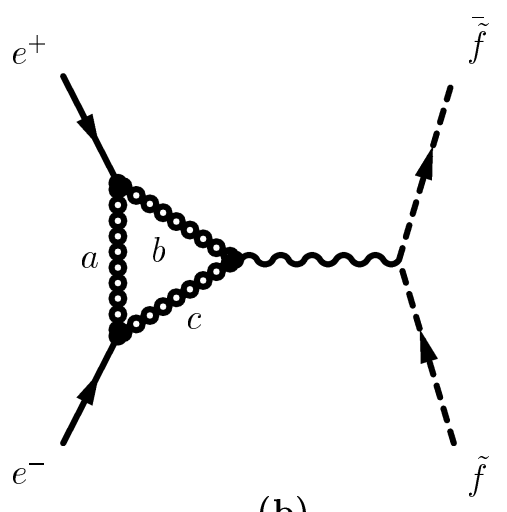

(b)

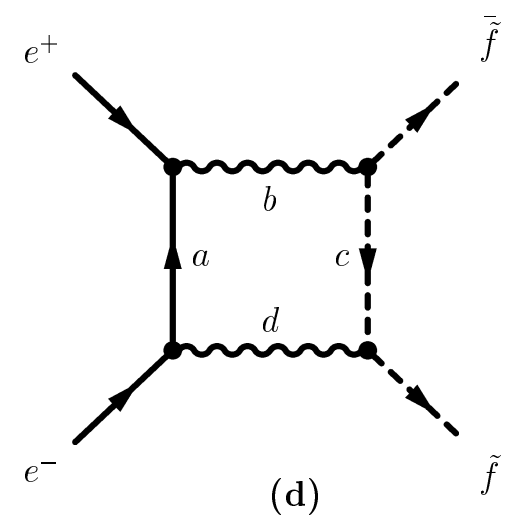

FIG. 2. Typical one loop diagrams for $e^{+} e^{-} \rightarrow \overline{\tilde{f}} \tilde{f}$ 


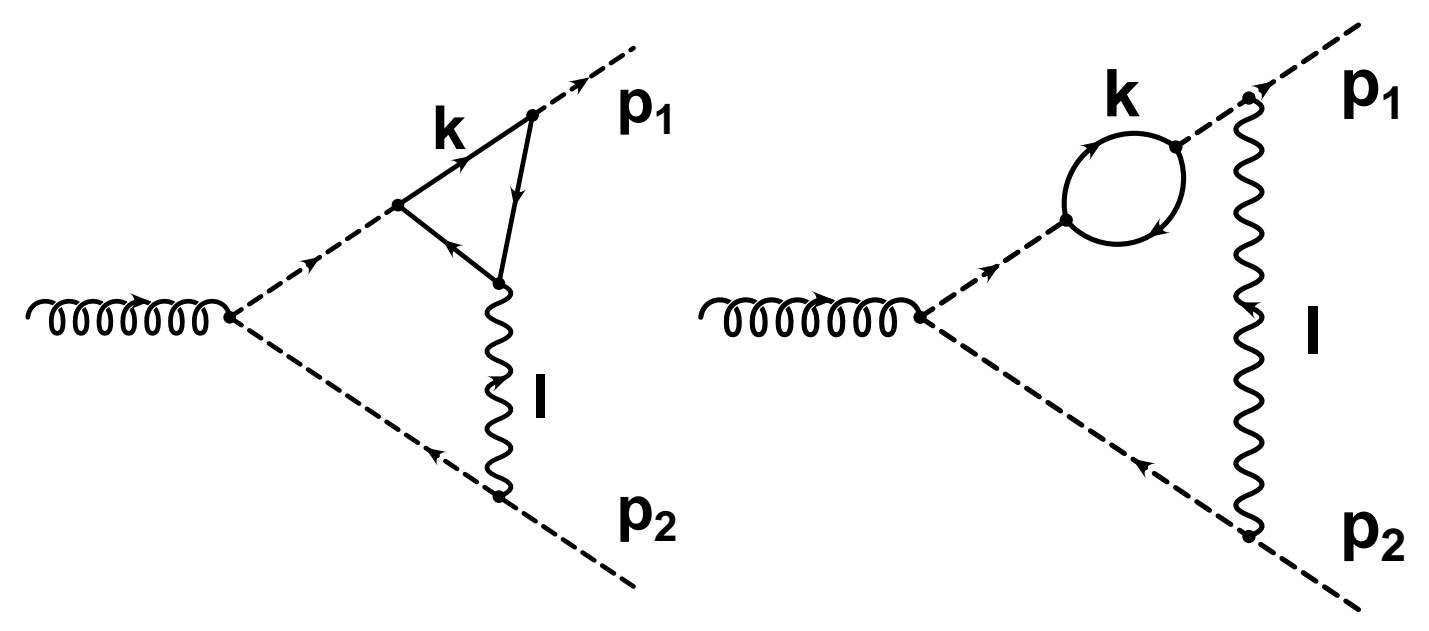

FIG. 3. Two loop corrections involving Yukawa couplings of scalars to fermions. The Ward identity in Eq. (3.5) assures that in the Feynman gauge, the sum of both diagrams does not lead to additional SL logarithms at the two loop level. Only corrections to the original one loop vertex (see section [II) need to be considered and lead to the exponentiation of Yukawa terms in the MSSM to SL accuracy. 


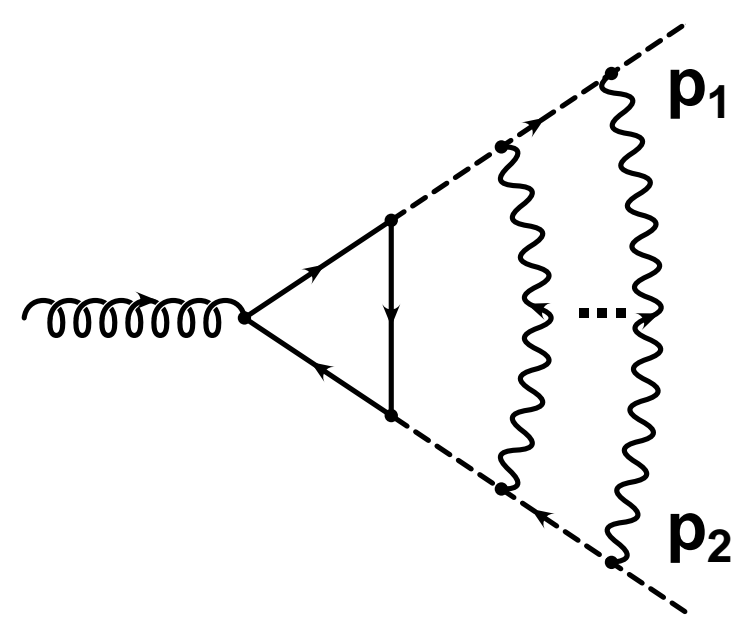

FIG. 4. Higher order corrections to vertices with Yukawa couplings to SL accuracy. The graph is only schematic since in principle the gauge bosons couple to all external legs in the process. Due to the discussion in the text the non-Abelian version of Gribov's factorization theorem can be employed in the context of the infrared evolution equation method. 

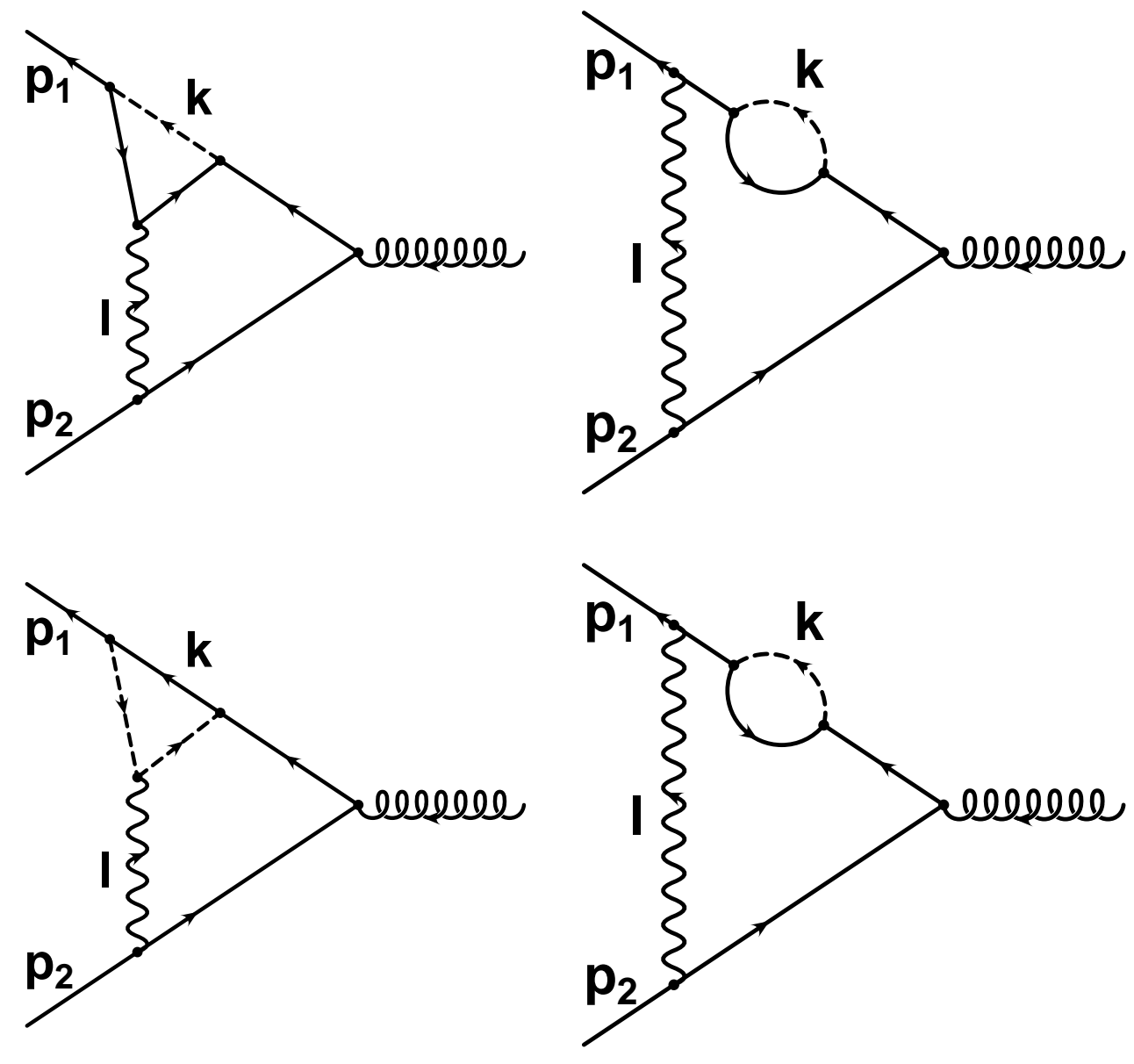

FIG. 5. Two loop corrections involving susy couplings of scalars to fermions. The Ward identity in Eq. (3.5) assures that in the Feynman gauge, the sum of both diagrams in each row does not lead to additional SL logarithms at the two loop level. Only corrections to the original one loop vertex (see section III) need to be considered and lead to the exponentiation of gauge terms in the MSSM to SL accuracy. 

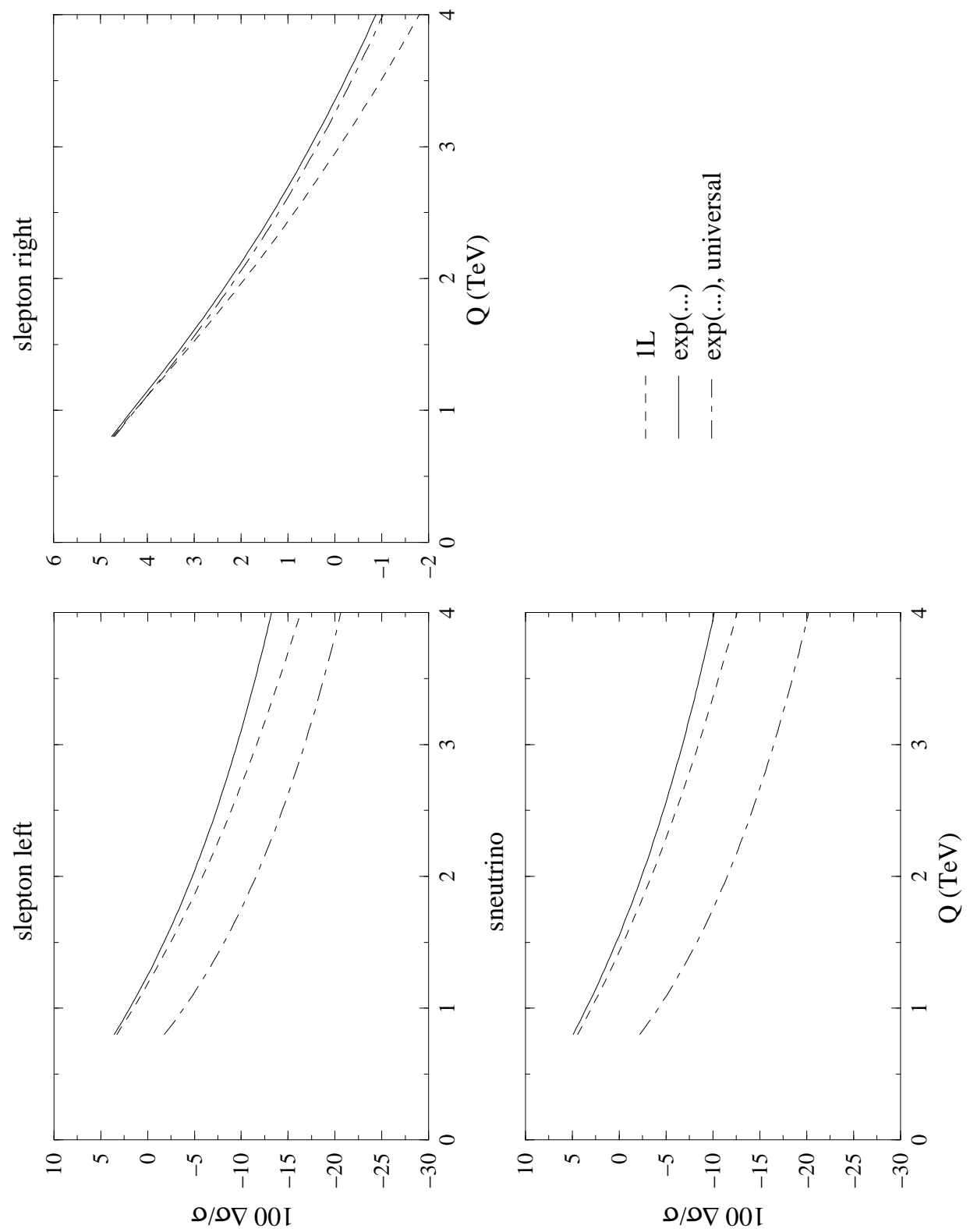

FIG. 6. Cross section for production of sleptons $(\neq \widetilde{e})$ or sneutrinos. "1L" is the total (Renormalization Group and Sudakov) one loop virtual effect. "exp(...)" is the cross section resummed at subleading order including RG contributions. In the last curve, labeled "exp(...), universal", the angular dependent terms coming from boxes have been suppressed. In this and the following Figures we denote by $Q$ the c.m. energy $\sqrt{q^{2}}$. 

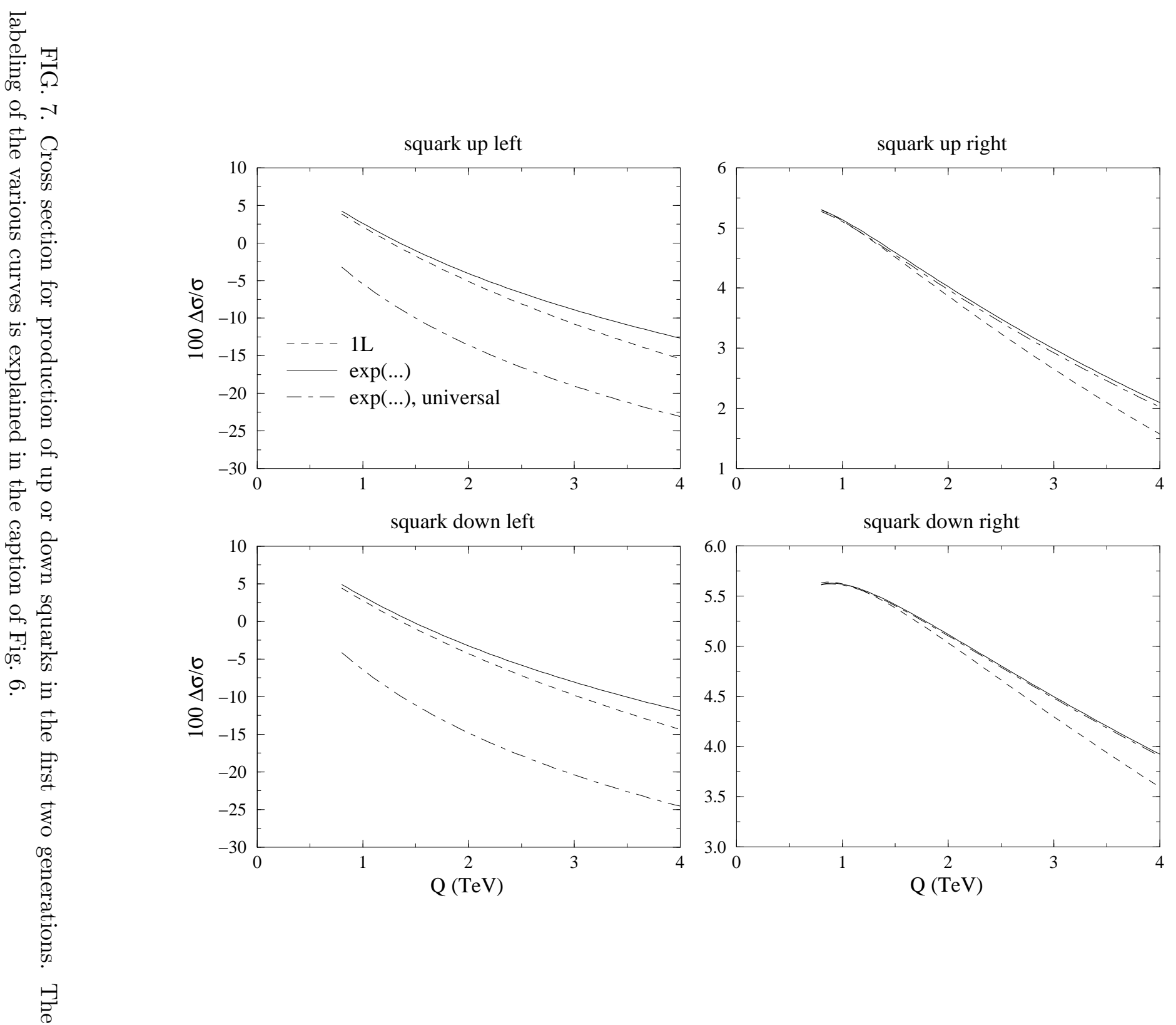

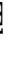



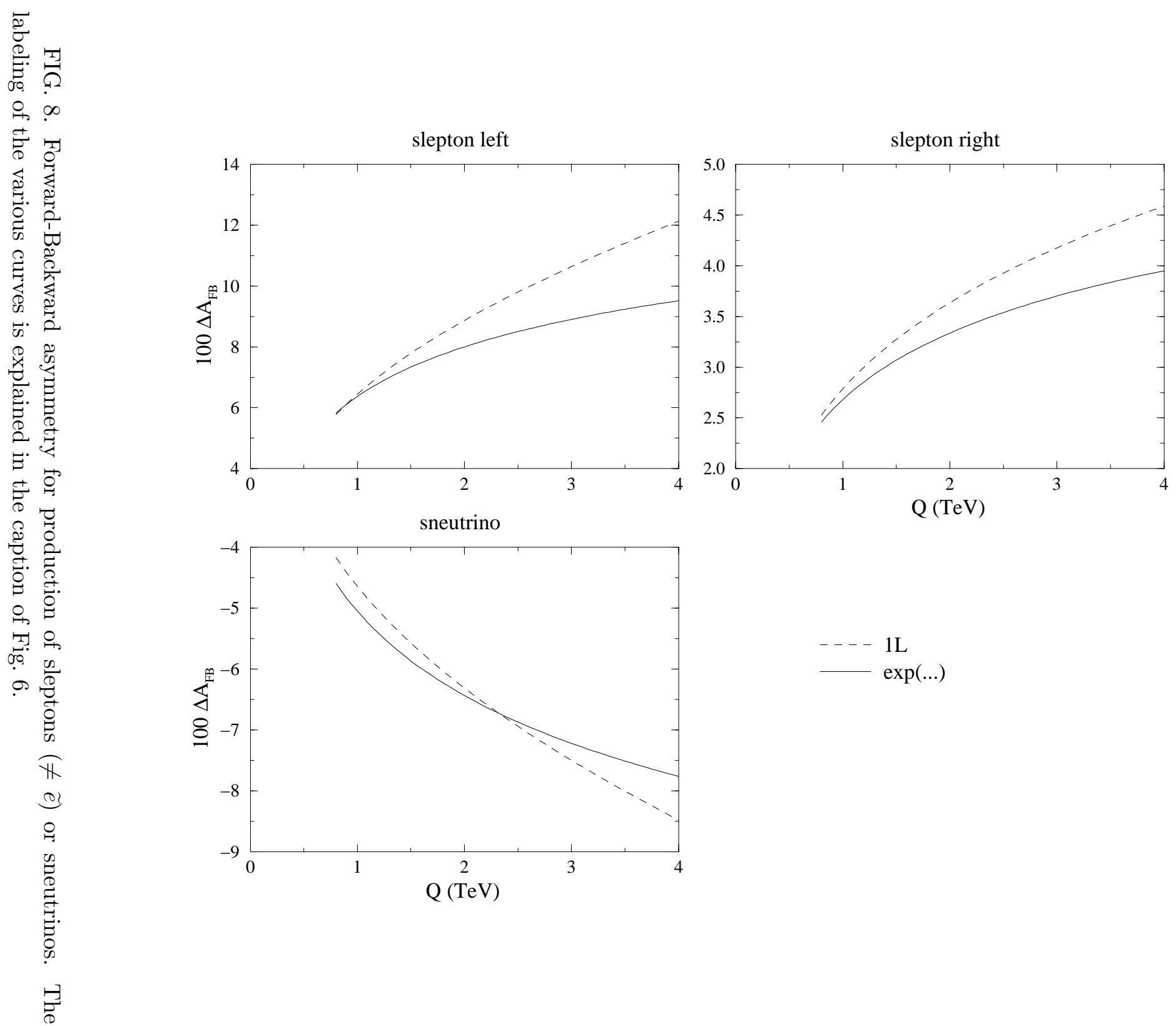

-.- 1L

$\exp (. .$. 

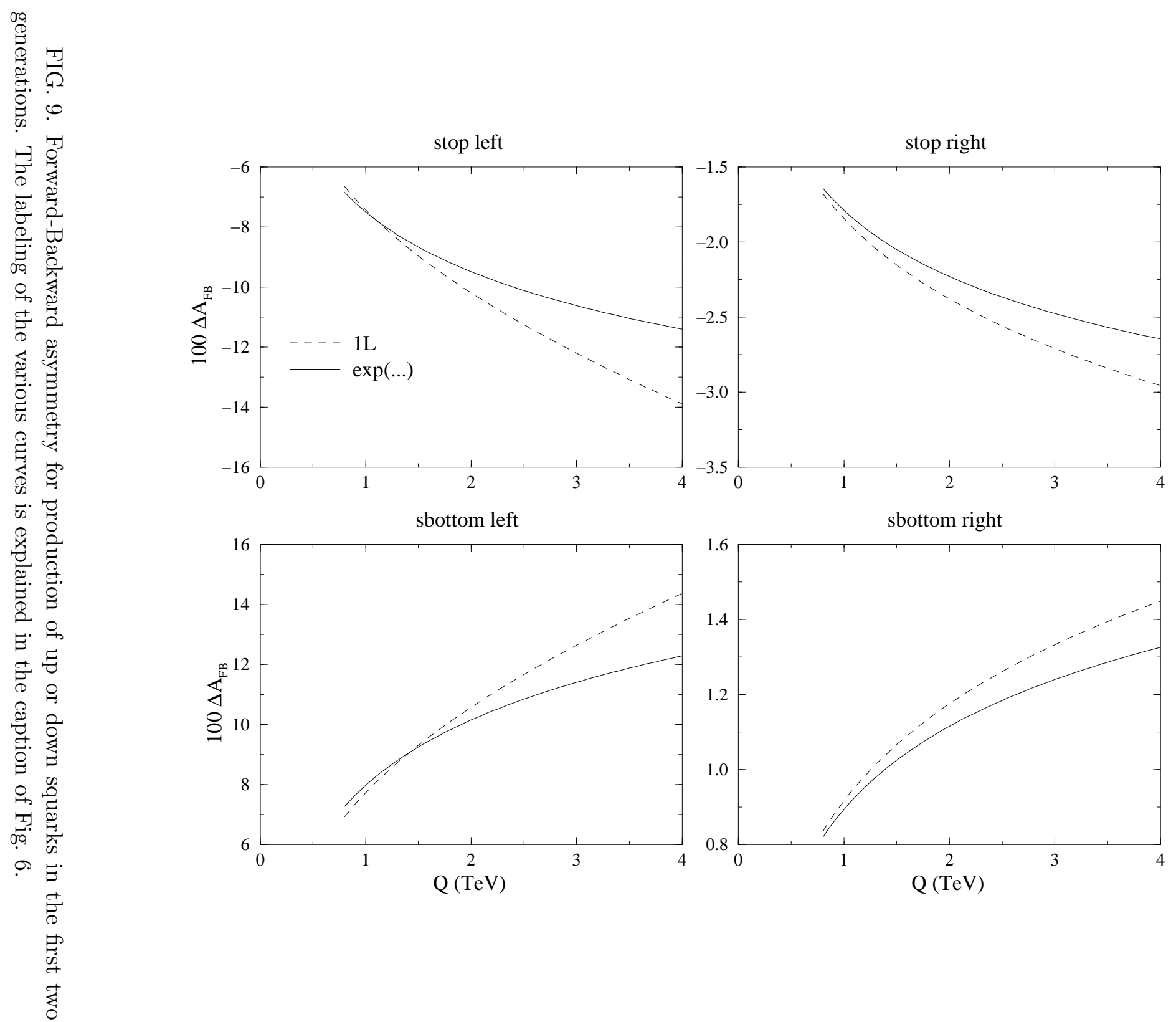

8

y 
stop left
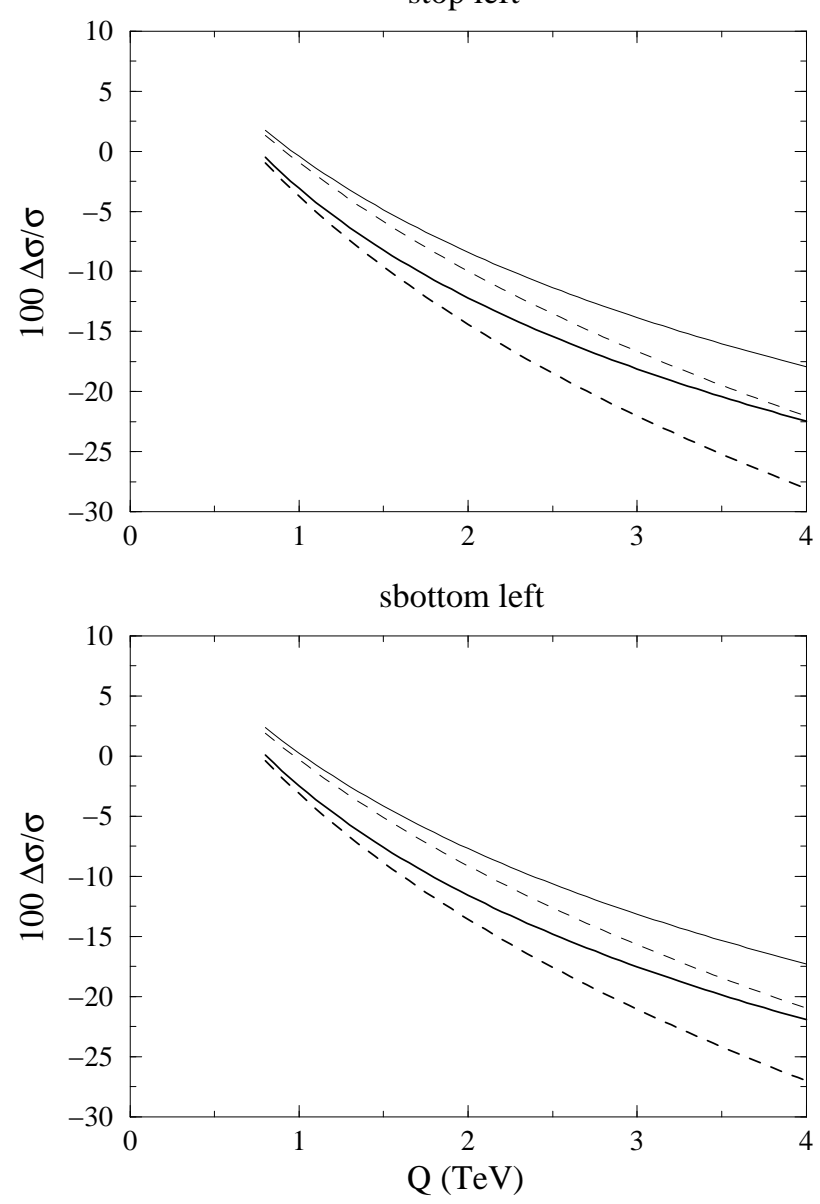

stop right
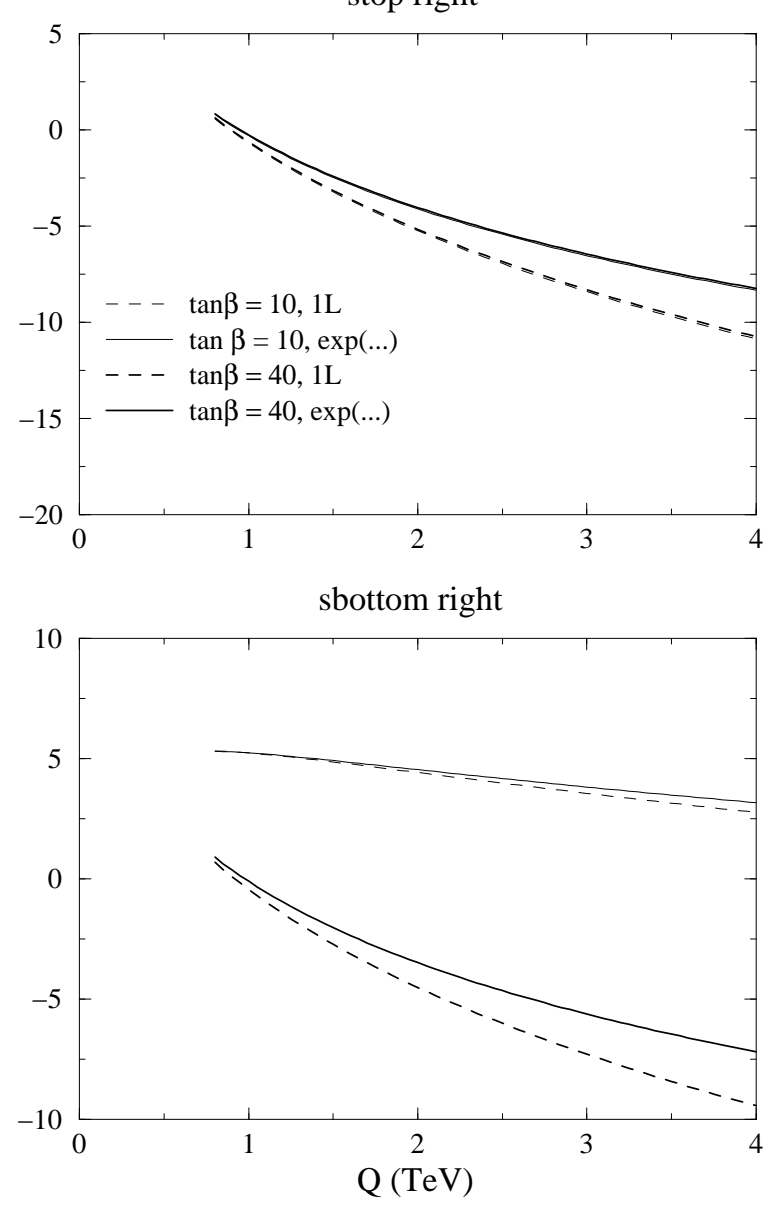

席 

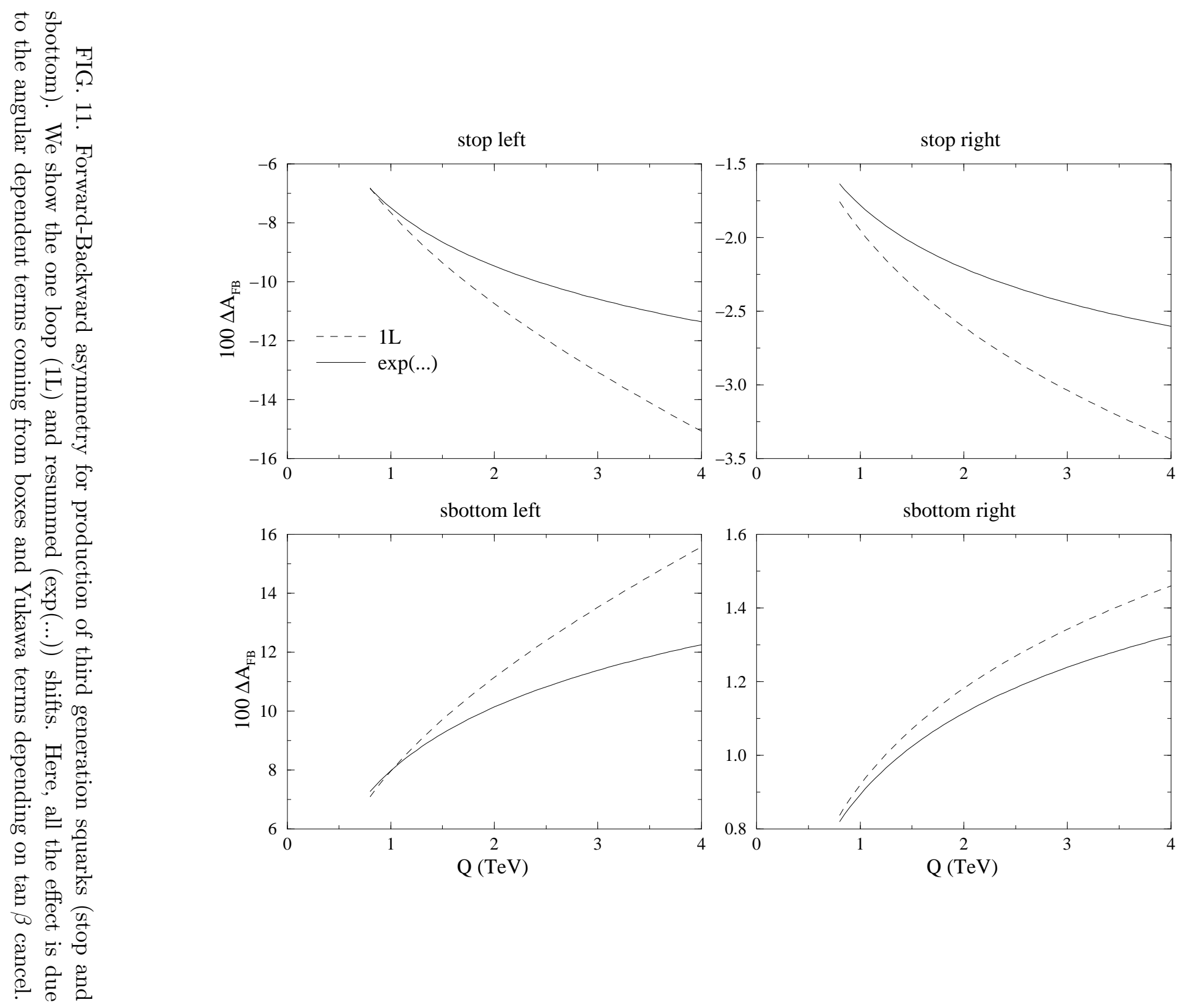

की

है

है 苍 

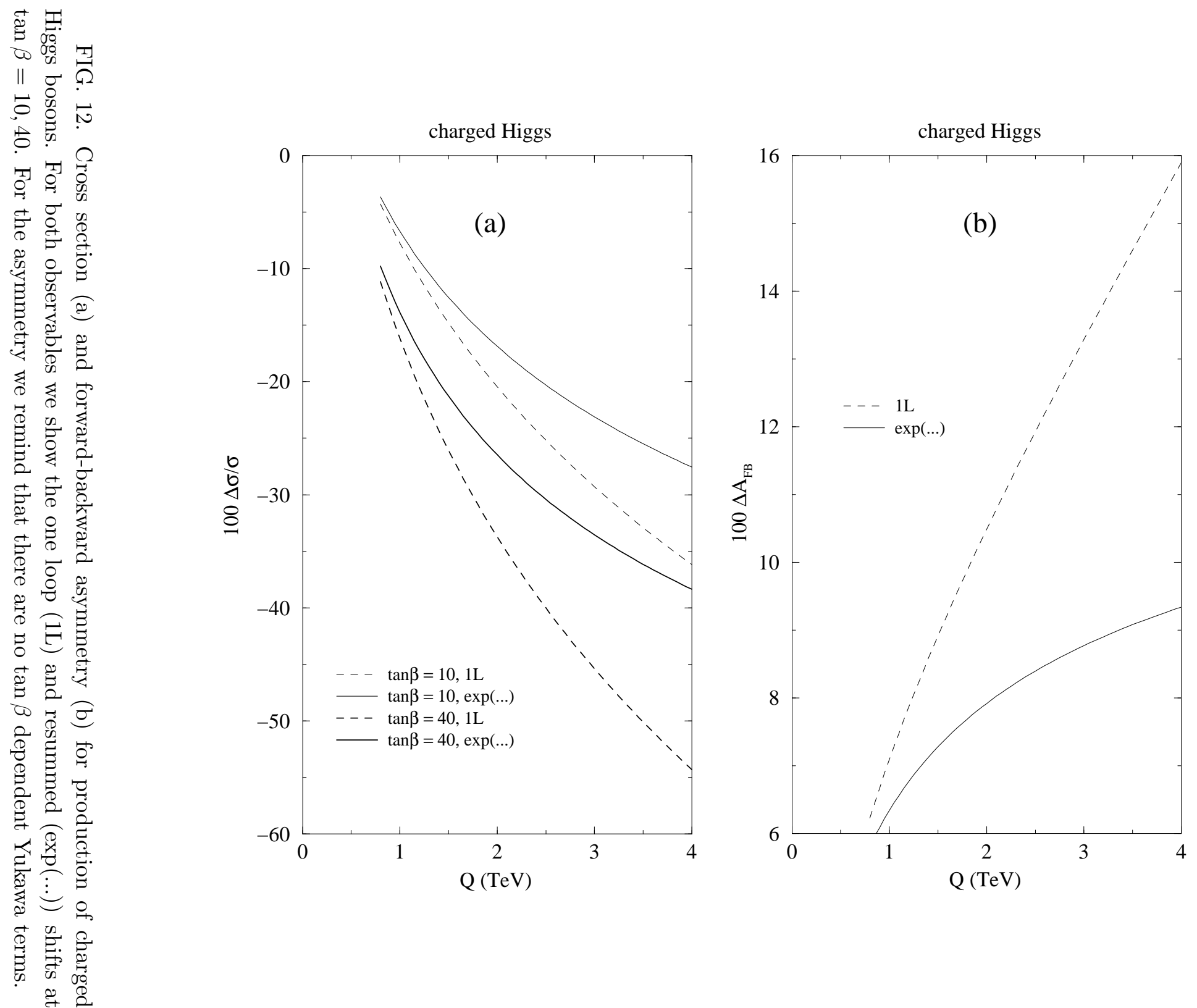


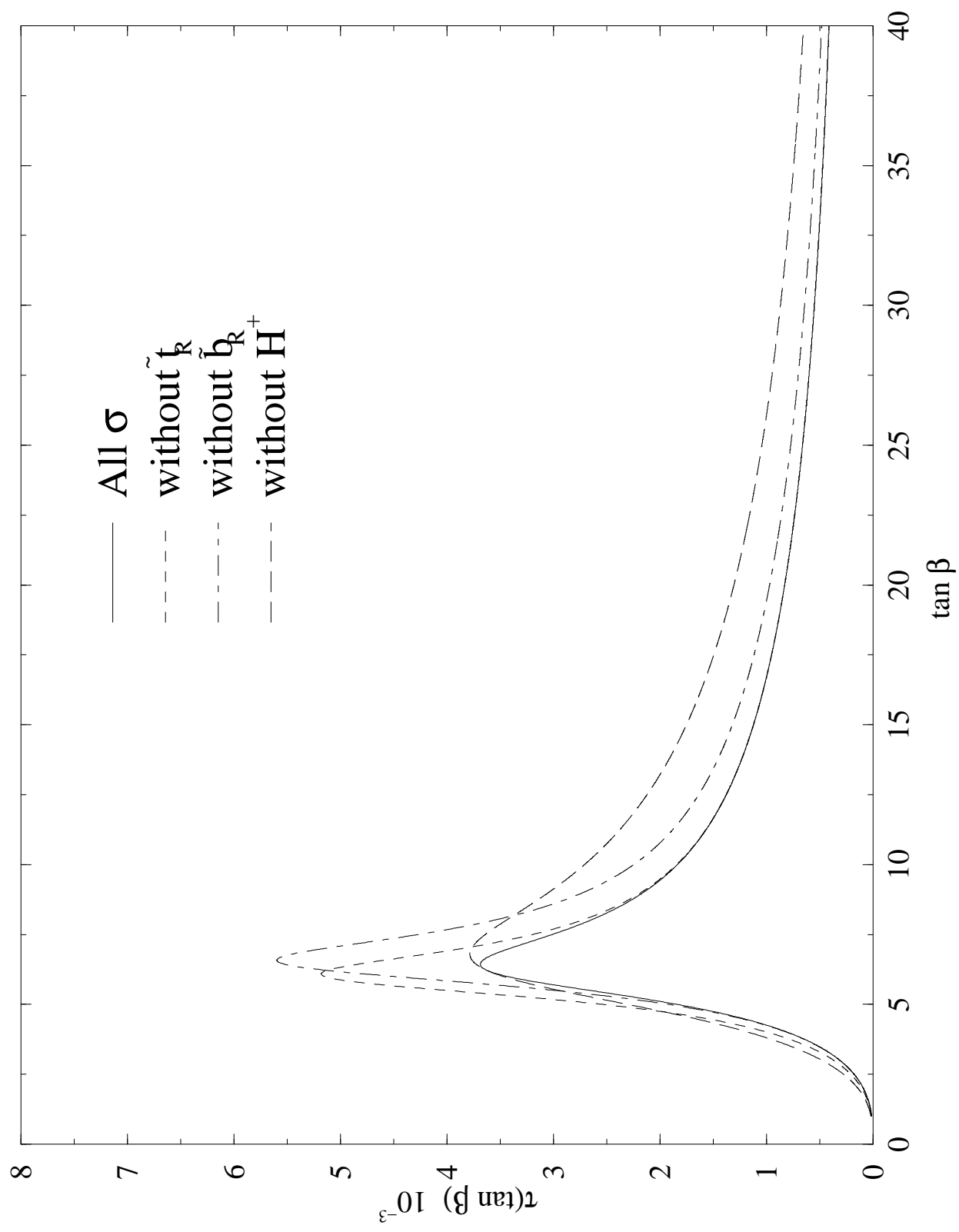

FIG. 13. This Figure shows the behavior of the $\tau$ function defined in Eq. (5.14). It increases when the slope of the SUSY effects does not depend much on $\tan \beta$. The four lines correspond to the four possible choices discussed in Sec. ( $\mathrm{V}$ ), that is: (i) all the five cross sections for production of third generation squarks and charged Higgs bosons, (ii) without production of $\widetilde{t}_{R}$, (iii) without production of $\widetilde{b}_{R}$, (iv) without production of charged Higgses $H^{ \pm}$. 


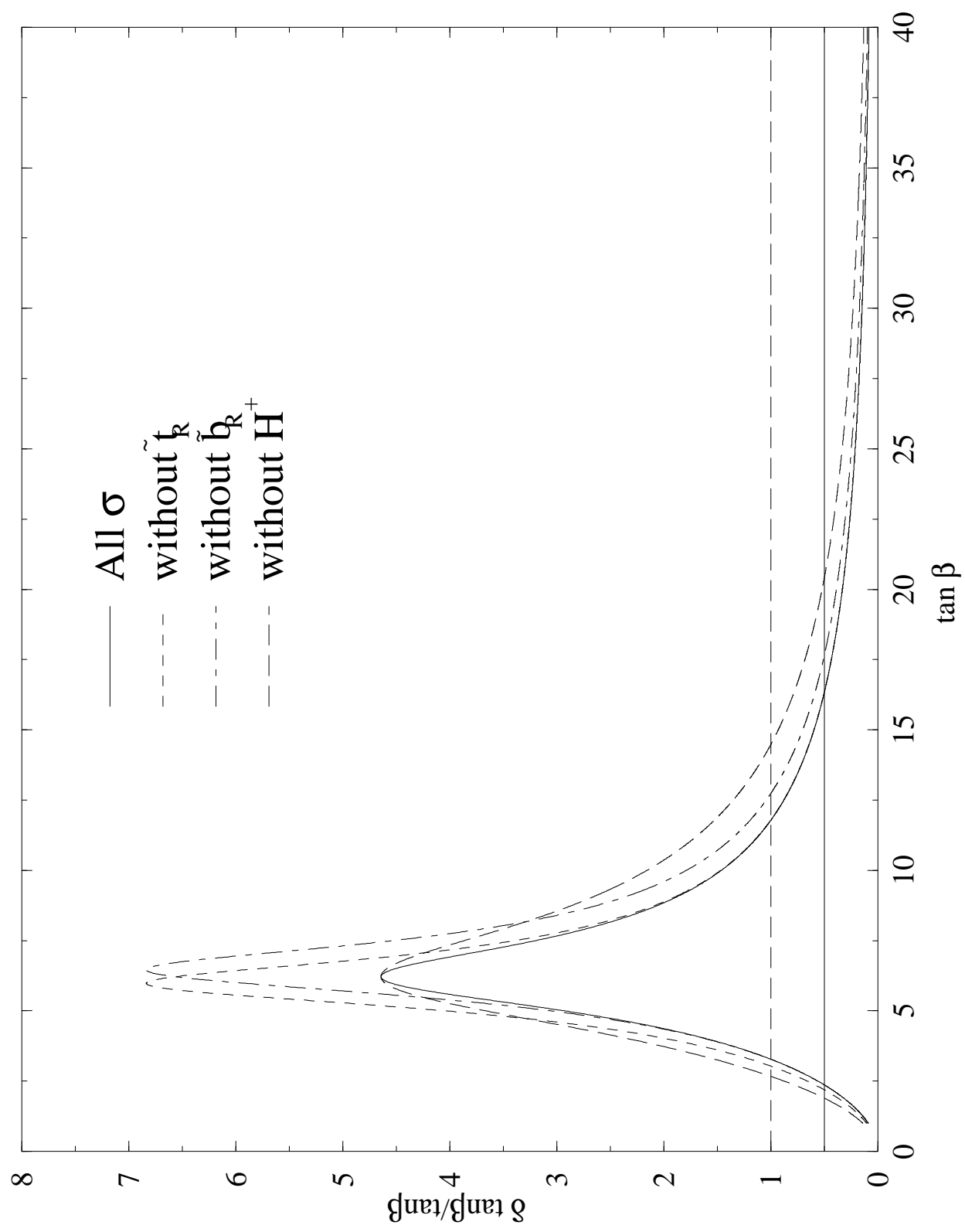

FIG. 14. We plot in this Figure the relative error $\delta \tan \beta / \tan \beta$ that can be derived in the determination of $\tan \beta$ assuming a relative accuracy of $1 \%$ on all the cross sections and the availability of 10 measurements at equally spaced energies between $800 \mathrm{GeV}$ and $1.5 \mathrm{TeV}$. for all the five observables. Again, we consider the optimal scenario when all the observables can be exploited as well as what happens when a subset of them is removed. In the best case, values of $\tan \beta>16$ can be determined with a $50 \%$ relative accuracy. 


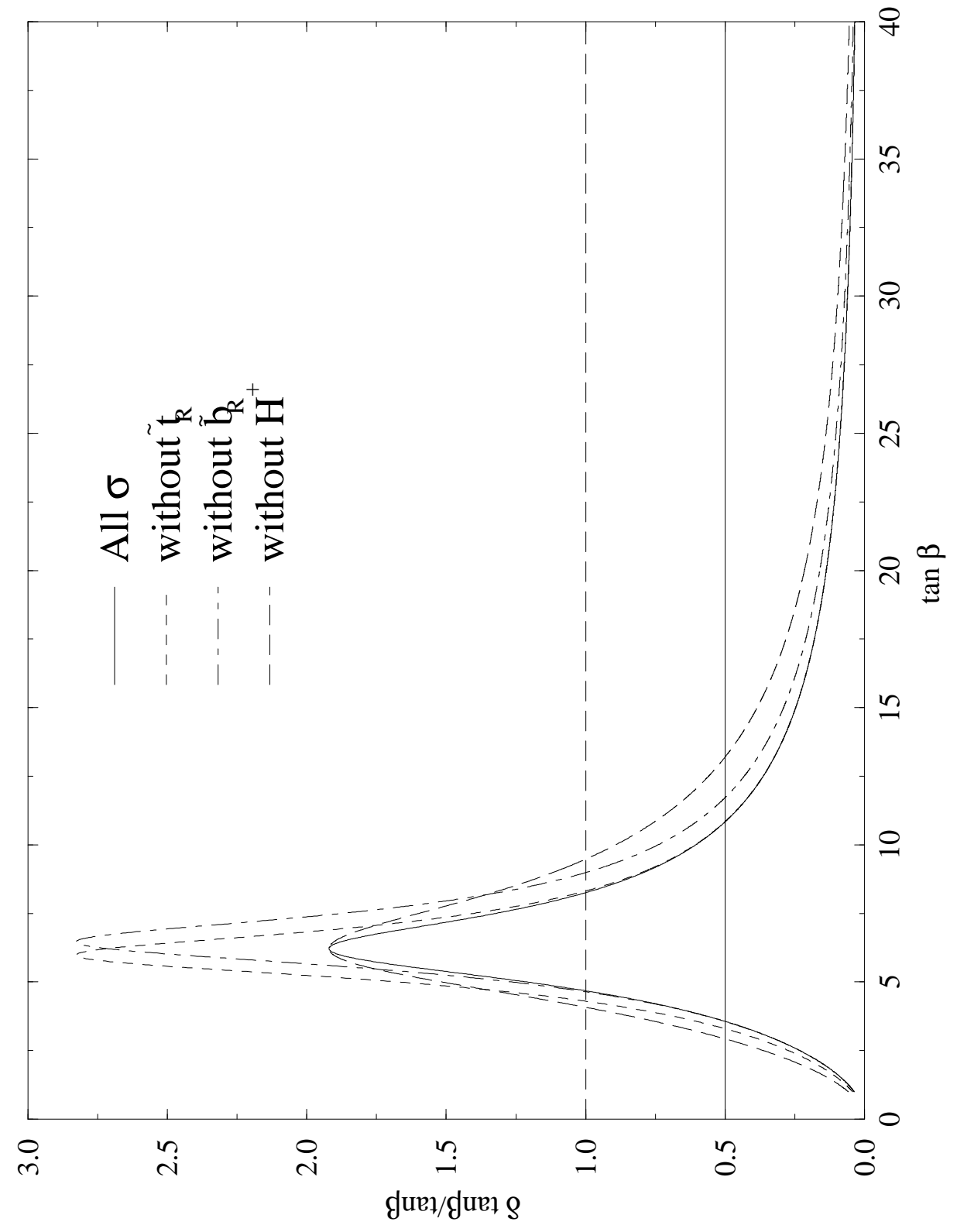

FIG. 15. Relative error $\delta \tan \beta / \tan \beta$ as in the previous Figure, but assuming the availability of 10 measurements at equally spaced energies between $800 \mathrm{GeV}$ and $3.3 \mathrm{TeV}$ (and again a relative $1 \%$ error on the measurements). In the best case, values of $\tan \beta>11$ can be determined if the relative accuracy is around $50 \%, \tan \beta>14$ if it is $25 \%$. 